Elsevier Editorial System(tm) for Nuclear

Inst. and Methods in Physics Research, A

Manuscript Draft

Manuscript Number:

Title: Computer Simulation tests of optimized neutron powder diffractometer configurations

Article Type: Full Length Article

Section/Category: Neutron Physics and Detectors

Keywords: neutron scattering; powder diffractometer; instruments; Monte Carlo simulation; McStas; Vitess

Corresponding Author: Dr. Leo Dominic Cussen, PhD

Corresponding Author's Institution:

First Author: Leo Dominic Cussen, PhD

Order of Authors: Leo Dominic Cussen, PhD; Klaus Lieutenant, Ph.D.

Abstract: Recent work has developed a new mathematical approach to optimally choose beam elements for constant wavelength neutron powder diffractometers. This article compares Monte Carlo computer simulations of existing instruments with simulations of instruments using configurations chosen using the new approach. The simulations show that large performance improvements over current best practice are possible. The tests here are limited to instruments optimized for samples with a cubic structure which differs from the optimization for triclinic structure samples. 


\section{Computer Simulation tests of optimized neutron powder 2 diffractometer configurations}

3 L. D. Cussen ${ }^{1}$ and K. Lieutenant ${ }^{2}$

$4 \quad{ }^{1}$ Cussen Consulting, 23 Burgundy Drive, Doncaster, 3108, Australia

5 Email: $\quad$ Leo@CussenConsulting.com

6 Telephone: $\quad+61401367472$

$7 \quad{ }^{2}$ Helmholtz Zentrum Berlin, Hahn-Meitner Platz 1, 14109 Berlin, Germany

$8 \quad$ Email: Klaus.Lieutenant@helmholtz-berlin.de

9 Abstract: Recent work has developed a new mathematical approach to optimally choose beam computer simulations of existing instruments with simulations of instruments using configurations chosen using the new approach. The simulations show that large performance improvements over current best practice are possible. The tests here are limited to instruments optimized for samples with a cubic structure which differs from the optimization for triclinic structure samples.

\section{Introduction}

Neutron powder diffraction is a valuable technique in studies of condensed matter. In comparison with other techniques, notably X-Ray diffraction, neutron diffraction has advantages in locating light atoms in crystal lattices and elucidating magnetic structures. The intensities in neutron scattering work are very low and so measurements are usually quite slow and are usually conducted with relatively poor resolution. In this context, it would be useful to improve the performance of neutron powder diffractometers (PDs).

Neutron powder diffractometers must distinguish the Bragg peaks scattered from a sample and this may be done using time-of-flight (TOF) methods (usually using a spallation neutron source) or using a crystal monochromator to produce a constant wavelength $(\mathrm{CW})$ beam (usually using a reactor source).

For CW PDs, one common instrumental arrangement is to have a primary spectrometer, which delivers a CW beam to the sample, followed by a sample and a collimator-detector pair which is stepped through a range of scattering angles, $2 \theta_{\mathrm{s}}$, to produce a map of scattered intensity as a function of $2 \theta_{\mathrm{s}}$. It is usual now to use a bank of many collimator-detector pairs to speed data collection. The primary spectrometer consists of the source and a crystal monochromator with beam collimators between source and monochromator and also between monochromator and sample. Assuming that the scattering plane is horizontal (as is usual), the monochromator is often vertically curved or "focussed" to increase vertical beam divergence and hence intensity at the sample. Many hope that horizontally curved monochromators may be exploited to further increase count rates by transforming beam spatial spread to angular spread thus increasing the flux at the sample position. A second common arrangement is to use an open geometry where the "banana" detector is a continuous multi-wire position sensitive detector (PSD). Collimated geometries have the advantages that noise tends to be low and that large samples can be used although, in practice, large samples are often simply unavailable. In any case, multiple scattering from the sample reduces the beam fraction scattered usefully and is a major contributor to background, so samples are usually chosen to scatter a maximum beam fraction of 1/e. Open geometries have the advantage of greatly increased count rates 
due to the larger effective detector solid angle but are sensitive to exact sample position and tend to be more susceptible to noise. Radial oscillating collimators between sample and detector are often used to reduce noise somewhat at a modest cost in count rate (of order 10\%).

Recent work [1,2] presents a new "Acceptance Diagram" approach to describing beams from primary spectrometers and an analytic approach to optimize the choice of beam elements for CWPDs. The "optimization" minimises the RMS value of $R_{\mathrm{P}}$, the "peak separation ability", at a fixed instrument transmission. $R_{\mathrm{P}}$ is the ratio of the Bragg peak angular widths $\left(A_{1 / 2}\right.$, the peak FWHM) to the expected separation of neighbouring peaks calculated from the peak density in reciprocal space for the sample type considered. For samples with cubic structures this is $R_{P}=A_{1 / 2} a_{0}^{2} \lambda^{-2} \sin 2 \theta_{S}$ where $a_{0}$ is the cubic unit cell side length. The optimization shows that at constant wavelength one can scale the values of beam collimations, mosaic and vertical divergence and that the instrument transmission, $\tau$, or the peak intensity, $L$, is then proportional to $A_{1 / 2}{ }^{4}$ or $R_{\mathrm{P}}{ }^{4}$. Any quality factor, $Q_{\mathrm{PD}}$, for the instruments must then include a $\tau / R_{\mathrm{P}}{ }^{4}$ term. For reference, a ${ }^{4} \sqrt{ } 10 \approx 1.8$ fold improvement in resolution at constant peak intensity is equivalent to a 10 fold increase in count rate at constant resolution. The optimization is less clear on the effect of wavelength but numerical tests seem to show that to measure some desired range of sample $d$-spacings, the wavelength should be made almost as long as is possible. The results of the optimization [2] suggest that a proper choice of elements can deliver large performance gains over current best practice, better described as reduced losses.

While the optimization mathematics is self-consistent and has been checked in several ways, some further independent verification would be useful. Some would say that the only true test of a prediction of instrument improvement is to build a new instrument and compare the data with that from existing machines. Such an exercise would cost many million Euros and permit testing only a single configuration. In the absence of a widely accepted expression for $Q_{\mathrm{PD}}$ for these measurements, it is surprisingly difficult to compare different instrument configurations unless the performance differences are truly dramatic. Significant work has been devoted to developing, testing, comparing and benchmarking "Monte Carlo" (MC) computer simulation packages for neutron scattering instruments, notably "McSTAS", "RESTRAX" and "VITESS" [3-5]. These programs have proved useful and cost effective in designing neutron scattering instruments. They provide a relatively cheap and quick way to accurately compare many different instrument configurations.

This article presents data from MC simulations of existing best practice CW PDs and of instrument configurations optimized using the new methods. The simulations were conducted in McSTAS (by LDC) and independently in VITESS (by KL) and then compared for consistency to provide an additional check of their validity. All figures (except figure 5) display the McSTAS data. The reference instruments here are taken to be the instruments D2B and D20 at the Institut LaueLangevin, highly regarded examples of so-called High Resolution and High Intensity PDs (HRPD \& HIPD).

Computer simulations such as those presented here cannot prove that the proposed new configurations are optimal so this article simply shows that the optimization procedure delivers significant performance improvements. There are an infinite number of optimal configurations for neutron CW PDs; even for those delivering particular resolution characteristics. There are many, many more non optimal configurations. This work should therefore be regarded as a preliminary and limited illustration of the improvements and possibilities offered. It is hoped that application of the new optimization method will lead to better instruments, better use of existing technology for instrument components, to better measurements, to new types of measurements and to other unexpected improvements. 
The Monte Carlo simulations are independent of the mathematical optimization so that even if the improved instrument configurations described here had been discovered by accident or guesswork they would still represent a significant and useful advance on current best practice. That the improvements have been found using a rational approach makes them more believable and useful.

\section{A baseline - simulations of existing instruments}

A list of symbols used for instrument parameters is presented in Appendix A. Appendix B presents details of the parameters used in each of the simulations which should allow the verification of the results presented here.

D2B is a conventional collimated high resolution CW PD using a bank of 128 detectors and $5^{\prime}$ FWHM collimators separated by $1.25^{\circ}$ thus spanning $160^{\circ}$ in $2 \theta_{\mathrm{S}}$. The instrument uses a $0.30 \mathrm{~m}$ high vertically focussed monochromator (VFM). There is some freedom in choosing the collimation between source and monochromator, $\left(\alpha_{1}=5^{\prime}, 10^{\prime}\right.$ or open $\left(\approx 22^{\prime}\right)$ FWHM) and a variable detector height, $2 H_{\mathrm{D}}$. The wavelength, $\lambda$, used is usually either $1.594 \AA$ or $2.4 \AA$. The two most commonly used configurations (E. Suard 2009 Private Communication) use $\alpha_{1}=5^{\prime}$, a germanium (Ge533) monochromator at Bragg angle $\theta_{\mathrm{M}}=67.5^{\circ}$ giving $\lambda=1.594 \AA$ and $2 H_{\mathrm{D}}=0.30 \mathrm{~m}$ or $0.10 \mathrm{~m}$.

D20 is a conventional open geometry CW PD using a large PSD with 1536 detector wires covering $153.6^{\circ}$ in $2 \theta_{\mathrm{S}}$. The McSTAS models here use 1601 wires over $160.1^{\circ}$. D20 has a number of available Bragg angles and monochromator crystal types, all vertically focussed, and the possibility to introduce a $10^{\prime}$ or $20^{\prime} \alpha_{1}$ collimator before the monochromator.

All simulations use a cylindrical sample of $\mathrm{Na}_{2} \mathrm{Ca}_{3} \mathrm{Al}_{2} \mathrm{~F}_{14}$ ("NAC") powder $8 \mathrm{~mm}$ in diameter and $20 \mathrm{~mm}$ high, unless otherwise stated. Note that the McSTAS PowderN sample component used does not model multiple scattering and has been used here with no incoherent scattering or absorption so there is no background in the simulations. The VITESS simulations include absorption in the sample. Multiple scattering is a major contributor to background on CW PDs. The monitors used in McSTAS simulations to represent detectors usually deliver the intensity as counts per second rather than the total counts at the detector. This has the benefit that when increasing the number of neutron packets simulated the statistical fluctuations reduce but the intensity does not change. When comparing data acquired from a scan using a single collimator and detector (or a multi-collimator multi-detector bank) with a scan acquired using a banana PSD, account must be taken of the different detector solid angles. In practice, a multi-detector bank must be stepped many times to complete a scan while a PSD takes a single measurement. For valid comparisons, the summed multi-detector bank intensities must be divided by the number of steps in the scan to account for the division of counting time. In the simulations described here, the number of steps is chosen to give complete angle coverage in the scan so for D2B the scans in the simulation are run using $15 \times 5$ ' steps. The data from each step are then summed and the totals divided by 15 to compensate for the division of total counting time needed. All McStas simulations assume a 100\% detector efficiency.

Figure 1 shows the simulated intensity as a function of scattering angle, $I\left(2 \theta_{\mathrm{S}}\right)$ for D2B as well as the calculated variation of angular resolution, $A_{1 / 2}\left(2 \theta_{\mathrm{S}}\right)$ and of $R_{\mathrm{P}}\left(2 \theta_{\mathrm{S}}\right)$. Figure 1a shows a simulation using Ge533 at $\theta_{\mathrm{M}}=67.5^{\circ}, \lambda=1.594 \AA, \alpha_{1}=5^{\prime}$ and $2 H_{\mathrm{D}}=0.10 \mathrm{~m}$. Figure $1 \mathrm{~b}$ uses the same parameters except that $2 H_{\mathrm{D}}=0.30 \mathrm{~m}$. Figure $1 \mathrm{c}$ shows a much less frequently used configuration where $\alpha_{1}=10$ ' and $2 H_{\mathrm{D}}=0.10 \mathrm{~m}$ which gives resolution almost identical to the usual high resolution mode and intensity comparable to the usual high intensity mode. The recognition that this particular configuration may be more useful came from a comparison of calculated values of $Q_{\mathrm{PD}}$ (taken to be $\tau / R_{\mathrm{P}_{-} \mathrm{RMS}}{ }^{4}$ ) for all D2B configurations. This illustrates that an established and accepted optimization 
process for neutron scattering instruments, especially if it includes a quantitative quality factor, can give information about the use of the machines as well as about their design and construction.

Values for $A_{1 / 2}\left(2 \theta_{\mathrm{S}}\right)$ and of $R_{\mathrm{P}}\left(2 \theta_{\mathrm{S}}\right)$ were extracted from the simulation output data, $I\left(2 \theta_{\mathrm{S}}\right)$, using simple statistical methods and found to be in close agreement to the calculated values in all cases discussed in this article except figure 5. Calculated values for D2B are illustrated in figures $1 \mathrm{~d}$ and $1 \mathrm{e}$.

A comparison between the illustrated data calculated using McSTAS and VITESS and real D2B data taken using a NAC sample shows extremely good agreement in peak shape except that the peaks at large $2 \theta_{\mathrm{S}}$ tend to be slightly lower and wider, no doubt due to Debye-Waller effect broadening from the room temperature sample which was not realistically modelled in these McSTAS simulations. The background present in the real data is also not present in simulated data but can be artificially reproduced by adding an incoherent cross section of about 200 barns to the sample in the McSTAS simulations. The close comparison gives confidence that these simulations are representative of actual instruments.

Figure 2 shows simulated data, $I\left(2 \theta_{\mathrm{S}}\right), A_{1 / 2}\left(2 \theta_{\mathrm{S}}\right)$ and $R_{\mathrm{P}}(\kappa)$, for D20 using a pyrolytic graphite monochromator (PG002) at $\theta_{\mathrm{M}}=-21^{\circ}$ and a germanium (Ge311) monochromator at $\theta_{\mathrm{M}}=-45^{\circ}$ both giving $\lambda=2.41 \AA$ and both used with an open beamtube before the monochromator giving $\alpha_{1} \approx 24^{\prime}$. A $0.05 \mathrm{~m}$ thick pyrolytic graphite filter was included in the model for the PG002 scan. Introducing an $\alpha_{1}$ collimator seems to make little difference to the simulated instrument resolution but reduces intensity greatly.

Note that for $\mathrm{D} 2 \mathrm{~B}$, where $\theta_{\mathrm{M}}$ is positive, the data ranges from $-10^{\circ}>2 \theta_{\mathrm{S}}>-170^{\circ}$ while for D20, where $\theta_{\mathrm{M}}$ is negative, the data ranges from $10^{\circ}<2 \theta_{\mathrm{S}}<170^{\circ}$. Note also that when the value of $R_{\mathrm{P}}$ exceeds 0.5 neighbouring Bragg peaks overlap. In practice, Rietveld analysis methods can be applied to extract useful data from overlapping peaks and overcome this problem to some extent.

TOF PDs on short pulse sources have the advantage that there is no real need to waste much source flux in monochromating the beam. CW PDs have the advantages that they can exploit wavevector focussing and vertical beam focussing both of which are more challenging to do with TOF machines. Stride et al. [6] used MC computer simulations to compare the performance of a CW PD and a Time-of-Flight (TOF) PD. They concluded that the performance of the TOF machine simulated was better than that of their reference CW PD by a factor of between 3 and 14 . The CW PD they modelled used distances of $10 \mathrm{~m}$ from source to monochromator, $2 \mathrm{~m}$ from monochromator to sample and $1.5 \mathrm{~m}$ from sample to detector. The source was $6 \times 10 \mathrm{~cm}^{2}$ source, $\alpha_{1}=\eta_{\mathrm{M}}=\alpha_{3}=12$ ' and they used a 20x20 $\mathrm{cm}^{2}$ VFM of $30 \%$ reflectivity. Figure 3 shows data from a McSTAS simulation modelling this CW PD. 61 detectors were modelled and stepped over $2.5^{\circ}$ in $30 \times 5^{\prime}$ steps. The simulated count rate here was divided by 30 to account for the time division between steps. Note that the monochromator reflectivity here is lower than the value of $40 \%$ assumed for germanium monochromators in the other simulations described here so the intensity was multiplied by $4 / 3$ to allow for this. The $85 \%$ detector collimator transmission is much higher than that used in the other simulations so the intensity has also been multiplied by $0.7 / 0.85$ to compensate for this.

Comparison with the performance of the optimized machines presented later seems to show that the optimized CW diffractometers are better than this design by more than a factor of 3-14. This result in no way proves that CW PDs are superior to TOF PDs in general but it does re-open the question. To answer that question would require a comparison between optimized CW PDs and TOF 
PDs. It appears likely that the method used to derive the CW PD optimization applied here could be adapted with some work to produce an optimization for TOF PDs.

\section{Optimized high resolution CW PD configurations using collimators}

In calculating optimized instrument parameters, there is considerable flexibility in the choice of primary spectrometer parameters to deliver a given beam, as discussed in [1] and [7]. For the optimized configurations discussed in this section, this flexibility has been used to maintain $\theta_{\mathrm{M}}=67.5^{\circ}$ and $\lambda=1.594 \AA$ to simplify comparisons to figure 1 . In general, for horizontally flat monochromators, $\theta_{\mathrm{M}}$ must be greater than $\theta_{\mathrm{SF}}$, the sample scattering angle where the angular resolution is best. By contrast, for concave horizontally focussed monochromators, $\theta_{\mathrm{M}}$ must be less than $\theta_{\mathrm{SF}}$. Using smaller values for $\theta_{\mathrm{M}}$ may offer some technical advantages. All optimizations discussed in this article assume that the sample has a cubic structure and that the scattering range of interest is $20^{\circ}<\left|2 \theta_{\mathrm{S}}\right|<160^{\circ}$. The optimization for triclinic structures is different to that for cubic structures and requires a larger value for $\theta_{\mathrm{SF}}$. The purpose of this work is to test if the optimization approach delivers improvements over current practice.

Figure 4 shows $I\left(2 \theta_{\mathrm{S}}\right), A_{1 / 2}\left(2 \theta_{\mathrm{S}}\right)$ and $R_{\mathrm{P}}(\kappa)$ for three optimized versions of $\mathrm{D} 2 \mathrm{~B}$ using collimators and horizontally flat VFMs. Figure 4a illustrates a high resolution configuration using the current detector collimation, $\alpha_{3}=5$ '. Note that the resolution here is markedly better than that in figure 1a. This better resolution configuration has reduced peak intensity. One expects intensity to scale as the 4-th power of resolution (the intensity loss here is smaller than that) and peak height to be proportional to intensity divided by peak width. Because of the better resolution, the peak heights here are quite comparable to those in figure 1a despite the better resolution and lower intensity.

Figure $4 \mathrm{~b}$ shows a high resolution configuration using $\alpha_{3}=7.94$ ' chosen to give an RMS value of $R_{\mathrm{P}}, R_{\mathrm{P} \_\mathrm{RMS}}$, equal to that for figure 1a. Thus, this configuration delivers resolution equivalent to the current highest resolution D2B configuration but with roughly four times the intensity. How does this arise? The optimization balances the resolution contributions from in plane beam divergence before and after the sample, vertical divergence before and after the sample and the wavelength spread. In this case, increasing the in-plane divergence and compensating by reducing vertical divergence one can obtain better transmission at the same resolution. In effect, one reduces the losses in the instrument. Significantly, the $\alpha_{3} \approx 8^{\prime}$ ' collimation used here is larger than the 6' collimation of the existing D20 PSD. That means that one could achieve better resolution than the current best on D2B using an open geometry CW PD and the additional solid angle coverage of the PSD would give a factor of 15 increase in count rate.

Figure $4 \mathrm{c}$ shows a higher intensity collimated configuration using 10' detector collimators. This represents the highest intensity optimized collimated configuration achievable on the D2B beamtube with $\theta_{\mathrm{M}}=67.5^{\circ}$; the limitation here is the natural collimation of the beam between source and monochromator. Clearly, it would be possible to increase that divergence using a guide between source and monochromator or reducing the distance between source and monochromator. It is also possible to adjust other parameters to optimally use a larger detector collimation but it is simpler to produce higher intensities using an open geometry and smaller $\theta_{\mathrm{M}}$ as discussed in the next section. The optimized configurations scale in $\alpha_{1}, \eta_{\mathrm{M}}, \alpha_{2}, \alpha_{3}, \sqrt{ } \phi_{2}$ and $\sqrt{ } \phi_{3}$ with intensity proportional to $\alpha_{3}{ }^{4}$ so that in principle and in simulations one can simply adjust the parameters to achieve the desired intensity-resolution trade-off for the problem under consideration. 


\section{An optimized high resolution CW PD using open geometry and a horizontally curved monochromator}

Figure 5 shows the VITESS simulated scan data for an optimized open geometry CW PD using a double focussed monochromator (DFM) curved both horizontally and vertically for comparison to D2B as described in $\S 2$ and the optimized collimated configurations described in $\S 3$. In these examples the geometry is constrained to use the existing D20 beamtube, monochromator position and PSD. These configurations use $\theta_{\mathrm{M}}=-45^{\circ}$ (an existing D20 Bragg angle). To facilitate comparison with the data from the optimized collimated machines, the wavelength is maintained at $\lambda=1.594^{\circ}$ in the simulations. A Ge511 monochromator crystal gives $\lambda=1.594 \AA$ at $\theta_{\mathrm{M}}=-47.06^{\circ}$ and $\lambda=1.54 \AA$ at $\theta_{\mathrm{M}}=45^{\circ}$ so the monochromator $d_{\mathrm{M}}$ value used in the simulations does not correspond exactly to any germanium Bragg peak: this is a minor detail. It is very expensive in intensity to improve resolution. Similarly it is expensive in intensity to increase the number of peaks in a scan.

Figure 5a shows a high resolution instrument, optimised to fully use the 6' detector collimation available using the D20 PSD. To achieve 6' collimation for $\alpha_{3}$ here requires restricting the sample diameter to $2.5 \mathrm{~mm}$ to match the detector wire spacing. This reduces the sample volume by more than a factor of 10 when compared to an $8 \mathrm{~mm}$ diameter sample but even so the count rate is increased by an order of magnitude when compared to D2B's usual high resolution mode.

Figure $5 \mathrm{~b}$ shows the same configuration with an $8 \mathrm{~mm}$ diameter sample which gives an effective value of about 12' for $\alpha_{3}$. The contribution to $A_{1 / 2}$ from $\alpha_{3}$ is only one of four contributing elements and so the effect of changing $\alpha_{3}$, even doubling it as here, is not extremely big.

Surprisingly, in the equivalent McSTAS simulations, changing the sample size had showed no noticeable effect on peak width although intensities increased by an order of magnitude. Given that such an effect is expected and is seen as expected in the VITESS simulations it seems that the McSTAS "PowderN" component used in all simulations here may have some issue in scattering virtual neutrons from the correct position in the sample. All other McSTAS simulations use an $8 \mathrm{~mm}$ diameter cylindrical sample $20 \mathrm{~mm}$ high.

\section{Optimized CW PDs using open geometry and horizontally curved monochromators}

Figure 6 shows data from McSTAS simulations of optimized open geometry CW PDs with varying resolution all using DFMs. In plane collimation is provided by two slits placed between the reactor face and the monochromator. This arrangement gives great flexibility in the instrument's resolution characteristics. The first slit acts as a virtual source and defines the angular width of the beam reaching each point at the monochromator. The horizontal monochromator focussing is arranged so that the monochromator focusses from the virtual source to the sample. A second slit just before the monochromator controls the beam width there and immediately after the monochromator and thus controls the beam angular width visible at the sample. Both of these slits could be very heavy (perhaps 0.2 or $0.5 \mathrm{~m}$ thick) to reduce the gamma ray and fast neutron flux before the monochromator and thus reduce the radiation load on the monochromator and its shielding and the resulting background. If the virtual source is placed on a rail to allow its position with respect to the monochromator to be varied, its position can then be used to adjust the detector scattering angle where the resolution is best, $2 \theta_{\mathrm{SF}}$, without adjusting the monochromator Bragg angle. The vertical beam divergence must also be adjusted to maintain an optimal configuration if the in plane divergences are varied and this can be done using slits before the monochromator and just in front of the detector bank. The monochromator here is Ge511 at $\theta_{\mathrm{M}}=-45^{\circ}$ giving $\lambda=1.54 \AA$. The three configurations simulated in figure 6 are the same machine with altered slit widths being the only changes. Here, the 
PSD detector has been simulated with 1601 wire over $160.1^{\circ}$. To match the highest resolution mode on D2B requires only 8' detector collimation so this could be accomplished with a smaller number of detector wires or a detector of smaller radius. Reducing the number of wires should reduce the cost of the detector electronics and increasing the wire spacing should reduce cross talk between channels. Of course, this would also increase the count rate in each detector bin.

The McSTAS simulations here show slightly higher peaks in figure 6a than the equivalent VITESS results, probably due to the McSTAS "PowderN" effects noted in $\S 4$ but figures $6 \mathrm{~b}$ and 6c show no such difference.

Many in the diffraction community accept a fundamental difference between HRPDs and HIPDs. The description of the optimization in [2] shows that changing the resolution-intensity tradeoff is simply a matter of scaling. These simulations demonstrate that it is possible to design an instrument giving great and simple flexibility in the resolution-intensity trade-off while simultaneously delivering count rates and resolution comparable to the best currently possible but requiring different machines and design philosophies. The increased count rates illustrated here arise from the combination of several effects which are better regarded as reducing losses than as enhancing intensity. These effects are

- Matching beam elements to each other (the optimization)

$$
(\times 1.5-\times 16 \text { or more })
$$

- Increased solid angle coverage by Position Sensitive Detector $(\times 15)$

- Generation of rectangular rather than triangular profiles for $\tau(\gamma) \quad$ (ideally $\times 2 \sqrt{2}$ )

- Removal of transmission losses in collimators $\quad(\approx \times 4)$

The beam paths on the existing instruments pass through air which reduces the neutron flux by about $6 \%$ per metre travelled. Evacuating these paths as much as is practicable or using ${ }^{4} \mathrm{He}$ filled flight tubes (beam loss $\approx 0.3 \%$ per metre) could provide a further count rate gain of about $\times 2.5$. This would not necessarily be an easy thing to do but the improved performance would probably repay the effort. This has not been simulated here.

\section{Optimizing for a small $2 \theta_{\mathrm{S}}$ range}

The instrument configurations discussed above were all designed to measure over a large range of $2 \theta_{\mathrm{S}}$ as would be the case if collecting a large number of peaks to determine a crystal structure. There are measurements where the interest is mainly in some small section of the full pattern; as, for example, when separating peak splitting due to some phase transformation. There are also dedicated instruments such as strain scanners which are designed specifically to measure over only a very small range of $2 \theta_{\mathrm{s}}$. It is possible to optimize a CW PD for such measurements.

Figure 7a shows the pattern for a CW PD optimized to measure between $80^{\circ}<2 \theta_{\mathrm{S}}<100^{\circ}$ using $\theta_{\mathrm{M}}=-45^{\circ}$ and $\lambda=1.594 \AA$. Figure $7 \mathrm{~b}$ shows the pattern for an instrument optimized to measure between $2 \AA<d_{\mathrm{S}}<3 \AA$ using $\theta_{\mathrm{M}}=-21^{\circ}$ and $\lambda=2.41 \AA$. Note the enhanced resolution and peak height in the region of interest. The drop lines in figures $6 \mathrm{c}$ and $6 \mathrm{~d}$ indicate the range over which optimum resolution is sought. These instrument configurations have not been optimized in detail for wavelength variation, which is needed to get the best results in these cases, but rather to use instruments easily adapted from existing machines. Figure 7 is merely an indication of the possibilities in this regard. Optimising over a smaller angular range and including a change in wavelength can produce even larger gains in both resolution and peak height although some 
challenges are likely to be met here as an instrument optimised for a very small $\theta_{\mathrm{S}}$ range needs a very wide beam at the monochromator which would make shielding and background problematic.

\section{A “Magnetic Materials Powder Diffractometer" - measurements at large $d_{s}$}

The intensity-resolution trade-off for optimized CW PDs simply results in a scaling of instrument angular divergences as the desired transmission changes. This means that any fundamental distinction in design between HRPDs and HIPDs disappears. There is, however, a distinct application for neutron CW PDs in investigating magnetic structures. Often, the unit cell of magnetic structures is quite large meaning that magnetic Bragg peaks are found at relatively small scattering vectors, $\kappa$, (ie small $2 \theta_{\mathrm{S}}$ ). In addition, magnetic form factors mean that magnetic peaks tend to become very weak or invisible at large $\kappa$. So, for magnetic studies the range of interest is often confined to small $\kappa$. Designing an instrument for such work may result in a resolution characteristic such as that illustrated in figure 2a for D20 using a PG002 monochromator where the resolution is very good at small $2 \theta_{\mathrm{S}}$ but poor at large $2 \theta_{\mathrm{S}}$ and $R_{\mathrm{P}}$ exceeds 0.5 (ie peaks are no longer resolved) if $2 \theta_{\mathrm{S}}>94^{\circ}$ (ie $\kappa>3.8 \AA^{-1}$ or $d_{\mathrm{S}}<1.65$ ). If interest is genuinely limited to small $\kappa$, then using long neutron wavelengths becomes a sensible option, particularly if the beam comes from a cold source which enhances the intensity delivered at long wavelengths. Then, relatively relaxed angular resolution is sufficient to separate peaks and can be used to increase count rates.

Based on these considerations, figure 8 shows an instrument designed for measurements limited to $\kappa<3.8 \AA^{-1}$. Following the principle adopted in this work that instruments should be feasible at existing facilities, the instrument simulated here uses the ILL horizontal cold source followed by an $\mathrm{m}=2$ neutron guide $16.3 \mathrm{~m}$ long, $6 \mathrm{~cm}$ wide and $20 \mathrm{~cm}$ high with the monochromator at a distance of $18.5 \mathrm{~m}$ from the source. The physical dimensions chosen mean that this instrument cannot use optimised element choices but even so it is apparent that the longer wavelength gives very large intensities and excellent peak separation in this situation.

The minimum resolution (maximum intensity) on an optimized CW PD at this position is limited by the $20 \mathrm{~cm}$ limit on monochromator height. Intensity increases as the 4-th power of resolution or the 8-th power of vertical divergence (ie monochromator height) and much higher intensity could be achieved by using a higher, narrower guide. Alternatively, the instrument could be optimized to deliver even better resolution. Notice that existing diffuse scattering diffractometers, which usually also allow polarisation analysis, have a configuration which seems to be well adapted to this application of studying magnetic Bragg peaks. The new optimization process used here can be applied unaltered to diffuse scattering diffractometers.

\section{Optimized CW PDs using a shaped mask to control the effective detector height}

It is usual on CW PDs to use a constant detector height at all scattering angles, $2 \theta_{\mathrm{S}}$. The expression for Bragg peak intensity on CW PDs contains a $1 /\left|\sin 2 \theta_{\mathrm{S}}\right|$ term to allow for the effect of fixed detector height intersecting a larger fraction of the Debye-Scherrer cones at the ends of a scan. As discussed in [2], the expressions for CW PD peak angular width, $A_{1 / 2}$, show that, if the detector height, $2 H_{\mathrm{D}}$, is independent of $2 \theta_{\mathrm{S}}$, then at small and large $2 \theta_{\mathrm{S}}$ the contribution to $A_{1 / 2}$ due to vertical divergence becomes proportional to $\left|\cot 2 \theta_{\mathrm{S}}\right|$ and diverges. Similarly, at large (but not small) $2 \theta_{\mathrm{S}}$ the in-plane contribution to $A_{1 / 2}$ also diverges (although less rapidly) in proportion to ( $\tan \theta_{\mathrm{S}}-\tan \theta_{\mathrm{SF}}$ ).

This suggests that if the detector height were reduced at small and large $2 \theta_{\mathrm{S}}$ - specifically if $2 H_{\mathrm{D}} \propto\left|\sin 2 \theta_{\mathrm{S}}\right|-$ then the resolution at the ends of the scans may be improved and the integrated peak intensity due to resolution should then be constant through the scan. Detailed calculations were undertaken to derive expressions for optimum beam element choices under this condition. 
Simulations of the diffraction patterns for optimized CW PDs where $2 H_{\mathrm{D}} \propto\left|\sin 2 \theta_{\mathrm{S}}\right|$ are illustrated in figure 9. The instrument modelled there is arranged as follows:

- A source

- A heavy slit (whose width, $2 W_{\mathrm{v}}$, and distance between source and monochromator, $L_{1 \_\mathrm{VH}}$, are variable) acting as a virtual source for horizontal monochromator focussing.

- A pair of heavy slits immediately preceding the monochromator to control beam width $\left(2 W_{\mathrm{M}}\right)$ and height $\left(2 H_{\mathrm{M}}\right)$ there.

- A segmented double focussing mosaic monochromator with variable Bragg angle, $\theta_{\mathrm{M}}$, variable vertical and horizontal curvature and ideally with a rather large mosaic (consistent with retaining good peak reflectivity). The monochromator is vertically focussed from source to sample and horizontally focussed from virtual source to sample.

- An open flight path to the sample at distance $L_{2}$

- A cylindrical sample

- A shaped slit immediately before the detector bank with height proportional to $2 H_{\mathrm{D}_{-} 90}\left|\sin 2 \theta_{\mathrm{S}}\right|$. In practice, this means the opening is a wedge segment of a sphere with variable opening height.

- A multi-wire position sensitive detector.

A radial oscillating collimator should be included between the sample and the detector bank and as much of the flight path as is possible should be evacuated or ${ }^{4} \mathrm{He}$ gas filled but these effects are not modelled here.

This arrangement gives great flexibility. The vertical divergence before and after the sample is controlled by the monochromator height, $2 H_{\mathrm{M}}$, and the detector height (specified by the value at $\left.2 \theta_{\mathrm{S}}=90^{\circ}, 2 H_{\mathrm{D}_{-} 90}\right)$. The monochromator Bragg angle, $\theta_{\mathrm{M}}$, determines the wavelength. The monochromator in-plane radius of curvature, $R_{\mathrm{MH}}$, set by the distance between monochromator and virtual source, $L_{1 \_\mathrm{VH}}$, in concert with $\theta_{\mathrm{M}}$ determines the scattering angle where the resolution is best, $2 \theta_{\mathrm{SF}}$. The ratio of the virtual source width, $2 W_{\mathrm{V}}$, to $L_{1_{-} \mathrm{VH}}$ determines the in-plane beam divergence at the monochromator $\left(\alpha_{1}\right)$ and the ratio of the monochromator's projected width, $2 W_{\mathrm{M}}$, to the distance between monochromator and sample, $L_{2}$, determines the in-plane beam divergence at the sample $\left(\alpha_{2}\right)$. The beam divergence at the detector is set by the detector wire spacing.

Detailed calculations show that using such a detector mask slightly improves the calculated quality factor $\left(Q_{\mathrm{PD}}=\tau / R_{\mathrm{P}}{ }^{4}\right)$ over that for detectors of constant height, although not by very much. The simulated data shows that this arrangement increases the useful range of peak separation in a scan at a cost in measured peak intensity near the ends of the scan (but recall that this is actually an equalising of peak intensities).

\section{Cross check between McSTAS and Vitess}

All simulations were performed by LDC using the McSTAS simulation package [3] and then independently repeated by KL using the VITESS package [5]. The data obtained, intensity as a function of scattering angle, were fully consistent for the two simulation sets except for the case of figure 5 as is discussed above. The directly accessible quantities, the total detector count rate, $I_{\text {Tot }}$, and the line widths, agree well with differences usually below $10 \%$. Better than $10 \%$ agreement for $I_{\text {Tot }}$ and $A_{1 / 2}$ cannot really be expected because of the different modelling of sample, monochromator and 
collimators in the two simulation packages. The simulation data were independently analysed using different evaluation routines. For the McSTAS data, the peak widths are described as the ratio of peak angular width to expected peak spacing, $R_{\mathrm{P}_{-} \mathrm{RMS}}$, as described above. For the VITESS data, the peak width was taken to be the arithmetic average of the peak angular widths.

Table 2 in appendix A presents these data as well as an estimate of a normalised figure-ofmerit, $Q_{\mathrm{PD}}$, for each simulation. Hewat (private communication) has suggested that a powder diffraction quality factor should be proportional to the number of peaks observed, $N_{\mathrm{L}}$. $I_{\text {Tot }}$ is related to the individual peak transmission, $\tau$, multiplied by $N_{\mathrm{L}}$ and so here, $Q_{\mathrm{PD}}$ is calculated as $I_{\mathrm{Tot}} / R_{\mathrm{P} \_ \text {RMS }}{ }^{4}$ for the McSTAS data and as $I_{\mathrm{Tot}} / A_{1 / 2 \_\mathrm{Av}}{ }^{4}$ for the VITESS data. In our view, the numerical $Q_{\mathrm{PD}}$ values should be treated with some caution but the pictures presented clearly show that large performance improvements over current best practice are possible.

\section{Discussion and Conclusion}

In comparing the scan data for the various configurations discussed above it should be borne in mind that improving resolution is usually very expensive in individual peak intensity. Increasing the number of peaks by reducing the wavelength requires improved angular resolution to maintain peak separation ability and divides the total scattered intensity (which is roughly constant) between a larger number of peaks; so this is also very expensive in individual peak intensity. The current difficulty in quantitatively comparing the quality of data taken at different wavelengths is one reason that the majority of new models simulated here used the same wavelength.

The optimization criterion used in designing the machines tested in this work was to minimise the RMS value of $R_{\mathrm{P}}$ at fixed integrated peak intensity (strictly instrument transmission) over a chosen scattering angle range, usually $20^{\circ}<\left|2 \theta_{\mathrm{S}}\right|<160^{\circ}$. The RMS value rather than the average or the maximum value of $R_{\mathrm{P}}$ was chosen simply to make the mathematics tractable. There may be some better optimization criterion.

The optimized configurations help to guide the choice of the best available technology. In particular, using a PSD detector gives a gain factor of $\times 15$ over the 128 detector bank modelled here because there is no need to divide the counting time to step the detector bank. Multi collimator detector systems are usually chosen to achieve high resolution for large samples but the simulations here confirm that very high resolution can also be achieved with a PSD. The high resolution option of the instrument described by figure 6a has about the same resolution as the current high resolution setting of D2B, figure 1a. Line widths below $0.25^{\circ}$ can easily be reached as shown in figures 6,7 and 9. As the resolution when using a PSD is limited by both the sample and detector pixel sizes, in this case to about $0.2^{\circ}$, higher resolution requires reducing the sample diameter which may reduce the intensity advantage of a PSD to some extent. Of course, being able to use smaller samples effectively is a great advantage for these instruments.

Matching all parameters in the diffractometer through the optimization yields further performance improvements. While the vertical monochromator curvature modelled here gives a gain factor of about $\times 4$ by comparison to a flat monochromator, the horizontal curvature used in many of the models does not really deliver flux gains; its role is to adjust the slope of angle-wavelength correlations in the beam at the sample and hence control the detector angle at which the resolution is best. Using slits rather than collimators to control beam divergence reduces transmission losses. Adding these effects to the reduced losses in a well balanced PSD PD seems to result in performance improvements of 2 orders of magnitude or more. As an example, the high resolution option shown in figure 6a has about the same resolution as the best D2B option (figure 1a) but more than 100 times the 
count rate. Another interesting result from this work is that the optimal detector height is smaller than seems to be usual practice.

The optimization itself only delivers a part (albeit a significant part) of the gain factors demonstrated. Further potential gains from reduced air scattering, rectangular profile beam angular transmission and removing lossy collimators have all been understood for some time. Better use of existing monochromator crystal types may give further gains. None of the simulations considered air attenuation on instruments which results in a loss in beam flux of approximately $6 \%$ per metre. Over the path from reactor face to detector on D2B $(15 \mathrm{~m})$ or D20 $(16.7 \mathrm{~m})$ this represents a loss of 2/3 of the flux. The potential gains from evacuating or arranging ${ }^{4} \mathrm{He}$ filled flight paths (where the losses are of order $0.34 \%$ per metre) are large. None of the simulations use a radial oscillating collimator which would be used in practice on open geometry PDs and would reduce count rates by of order $10 \%$. Clearly, improved beamtube design and shorter source-monochromator distances or guides could offer additional gains.

Very large improvements in neutron CW PD performance appear to be possible. Obviously, this would permit better measurements to be conducted faster or to obtain good data from smaller samples. Very rapidly acquired patterns should permit chemical reactions to be studied in real time. The most exciting new possibilities are probably difficult to imagine now and would only become apparent from using better machines. The gain factors demonstrated above are almost certainly really achievable given that the configurations were derived mathematically and tested by calculation before being tested using MC computer simulations which completely confirm the predictions.

Acknowledgement: We thank Emmanuel Farhi for useful discussions, for his long term encouragement and for sharing information concerning instruments and simulation methods.

\section{References}

[1] Cussen, L.D. "A 2D Acceptance Diagram Description of Neutron Primary Spectrometer Beams" Submitted to Nucl. Instrum. Methods Nov. 2015

[2] Cussen, L.D. "Optimizing Constant Wavelength Neutron Powder Diffractometers" Submitted to Nucl. Instrum. Methods Nov. 2015

[3] Willendrup, P., Farhi, E. \& Lefmann, K. Physica B 350 (2004) 735

[4] Saroun, J. \& Kulda, J. Physica B 234-236 (1997) 1102-1104

[5] Lieutenant, K., Zsigmond, G., Manoshin, S., Fromme, M., Bordallo, H. N., Champion, J. D. M., Peters, J., Mezei, F., Proc. of SPIE 5536, ed. M. Sanchez del Rio (2004) 134 - 145

[6] Stride, J.A.; Wechsler, D.; Mezei, F.; Bleif, H-J. Nucl. Instrum. Methods A 451 (2000) 480491

[7] Cussen, L.D. Nucl. Instrum. Methods A554 (2005) 406-414

\section{Figure Captions}

Figure 1 D2B with $\lambda=1.594 \AA$, NAC sample $8 \times 20 \mathrm{~mm}^{2}$
(a) $I\left(2 \theta_{\mathrm{S}}\right)$ for $\alpha_{1}=5,2 H_{\mathrm{D}}=0.10 \mathrm{~m}$
(b) $I\left(2 \theta_{\mathrm{S}}\right) \alpha_{1}=5,2 H_{\mathrm{D}}=0.30 \mathrm{~m}$
(c) $I\left(2 \theta_{\mathrm{S}}\right)$ for $\alpha_{1}=10^{\prime}, 2 H_{\mathrm{D}}=0.10 \mathrm{~m}$
(d) $A_{1 / 2}\left(2 \theta_{\mathrm{S}}\right)$ and (e) $R_{\mathrm{P}}\left(2 \theta_{\mathrm{S}}\right)$, the "peak resolving ability"

Solid, dashed and dotted lines correspond to a, b and c respectively

Figure 2 D20 at $\lambda=2.41 \AA$, NAC sample $8 \times 20 \mathrm{~mm}^{2}$ 
Figure 3 Results of a simulation of the CW PD configuration used by Stride et al. for comparison with TOF PDs.

(a) $I\left(2 \theta_{\mathrm{S}}\right)$

(b) $A_{1 / 2}\left(2 \theta_{\mathrm{S}}\right)$

(c) $R_{\mathrm{P}}\left(2 \theta_{\mathrm{S}}\right)$.

Figure 4 Optimized collimated CW PDs based on D2B using VFMs at $\lambda=1.594 \AA$
(a) $I\left(2 \theta_{\mathrm{S}}\right) \alpha_{3}=5$
(b) $I\left(2 \theta_{\mathrm{S}}\right) \alpha_{3}=7.94$
(c) $I\left(2 \theta_{\mathrm{S}}\right) \alpha_{3}=10$ '

480

(d) $A_{1 / 2}\left(2 \theta_{\mathrm{S}}\right)$ - solid, dashed and dotted lines correspond to (a), (b) and (c)

(e) $R_{\mathrm{P}}\left(2 \theta_{\mathrm{S}}\right)$ - solid, dashed and dotted lines correspond to (a), (b) and (c)

Figure 5 Optimized open geometry high resolution CW PDs using DFMs.

Figure 6 Optimized open geometry CW PDs using DFMs and an $8 \mathrm{~mm}$ diameter NAC sample.

\begin{tabular}{|l|l|l|l|}
\hline$L_{1}, L_{2}, L_{3}$ & $\begin{array}{l}\text { Distance Source-Monochromator, } \\
\text { Monochromator-Sample, }\end{array}$ & $\begin{array}{l}2 H_{\mathrm{M}}, \\
2 H_{\mathrm{S}}, \\
\end{array}$ & $\begin{array}{l}\text { Full height of monochromator, } \\
\text { Sample, detector }(\mathrm{m})\end{array}$ \\
\hline$L_{1 \_\mathrm{VV}}$, & Distance Monochromator to & $R_{\mathrm{DH}}$, & \\
$L_{1 \_\mathrm{VH}}$ & virtual source for vertical, & $R_{\mathrm{MV}}$ & $\begin{array}{l}\text { Monochromator radius of curvature } \\
\text { (horizontal, vertical) }\end{array}$ \\
\hline
\end{tabular}




\begin{tabular}{|c|c|c|c|}
\hline & horizontal focussing $(\mathrm{m})$ & & \\
\hline$\theta_{\mathrm{M}}$ & $\begin{array}{l}\text { Monochromator Bragg Angle } \\
\text { (degrees) }\end{array}$ & $2 W_{\mathrm{VH}}$ & $\begin{array}{l}\text { Full width of virtual source for } \\
\text { horizontal focussing }(\mathrm{m})\end{array}$ \\
\hline$\theta_{\mathrm{S}}, 2 \theta_{\mathrm{S}}$ & $\begin{array}{l}\text { Bragg and scattering angles at } \\
\text { sample (degrees) }\end{array}$ & $2 W_{\mathrm{M}}$ & $\begin{array}{l}\text { Full horizontal width of slit } \\
\text { preceding monochromator (m) }\end{array}$ \\
\hline$\theta_{\mathrm{SF}}$ & $\begin{array}{l}\text { Sample scattering angle at which } \\
\text { the resolution is best }\end{array}$ & $2 W_{\mathrm{S}}$ & Full width of sample (m) \\
\hline$\lambda$ & Neutron Wavelength $(\AA)$ & $\kappa$ & Neutron Wavevector $\left(\AA^{-1}\right)$ \\
\hline$\alpha_{1}$ & $\begin{array}{l}\text { Angular width (FWHM) of } \\
\text { collimator between source and } \\
\text { monochromator }\end{array}$ & $\beta, \eta_{\mathrm{M}}$ & Monochromator crystal mosaic \\
\hline$\alpha_{2}$ & $\begin{array}{l}\text { Angular width (FWHM) of } \\
\text { collimator between } \\
\text { monochromator and sample }\end{array}$ & $\pm \phi_{2}$ & $\begin{array}{l}\text { Vertical beam divergence between } \\
\text { monochromator and sample }\end{array}$ \\
\hline$\alpha_{3}$ & $\begin{array}{l}\text { Angular width (FWHM) of } \\
\text { collimator between sample and } \\
\text { detector }\end{array}$ & $\pm \phi_{3}$ & $\begin{array}{l}\text { Vertical beam divergence between } \\
\text { sample and detector }\end{array}$ \\
\hline$R_{\mathrm{M}}$ & Monochromator peak reflectivity & $\bar{\tau} \tau_{\mathrm{i}}$ & Peak transmission of i-th collimator \\
\hline $\bar{\tau}$ & Transmission & $A_{1 / 2}$ & Angular resolution width in $2 \theta_{\mathrm{S}}$ \\
\hline$R_{\mathrm{P}}$ & $\begin{array}{l}\text { "Peak resolving power" }=A_{1 / 2} \times \\
\text { peak angular density in } 2 \theta_{\mathrm{S}}\end{array}$ & $Q_{\mathrm{PD}}$ & $\begin{array}{l}\text { "Quality Factor" here usually } \\
\text { Intensity / Resolution }{ }^{4}\end{array}$ \\
\hline$\overline{\alpha_{\text {In }}}$ & $\begin{array}{l}\text { Angular width (FWHM) of beam } \\
\text { at sample }\end{array}$ & & \\
\hline
\end{tabular}

503

504

\begin{tabular}{|c|c|c|c|c|c|c|c|c|c|}
\hline \multirow{2}{*}{\multicolumn{2}{|c|}{$\begin{array}{l}\text { Figure Description } \\
\text { Collimated machines }\end{array}$}} & \multirow[t]{2}{*}{$\lambda(\AA)$} & \multirow{2}{*}{$\begin{array}{l}\text { No. } \\
\text { Peaks }\end{array}$} & \multicolumn{3}{|c|}{ McSTAS } & \multicolumn{3}{|c|}{ VITESS } \\
\hline & & & & $I_{\mathrm{Tot}}$ & $R_{\mathrm{P} \_\mathrm{RMS}}$ & $\begin{array}{l}Q_{\text {Norm }}= \\
I_{\text {Tot }} / R_{\mathrm{P}}{ }^{4}\end{array}$ & $I_{\text {Tot }}$ & $A_{1 / 2 \_\mathrm{Av}}$ & $\begin{array}{l}Q_{\text {Norm }}= \\
I_{\text {Tot }} / A_{1 / 2}{ }^{4}\end{array}$ \\
\hline $1 \mathrm{a}$ & D2B 5'/10 cm & 1.594 & 76 & 100 & 0.152 & 6.1 & 90 & 0.312 & 3.3 \\
\hline $1 b$ & D2B 5'/30 cm & 1.594 & 76 & 313 & 0.318 & 1.0 & 270 & 0.553 & 1.0 \\
\hline $1 \mathrm{c}$ & D2B 10' $10 \mathrm{~cm}$ & 1.594 & 76 & 220 & 0.158 & 11.5 & 201 & 0.33 & 5.9 \\
\hline 3 & Stride et al. & 1.50 & 84 & 940 & 0.245 & 8.5 & 1257 & 0.521 & 5.9 \\
\hline $4 \mathrm{a}$ & Opt 1 & 1.594 & 76 & 48.6 & 0.096 & 18.7 & 45 & 0.202 & 9.4 \\
\hline $4 b$ & Opt 2 & 1.594 & 76 & 429 & 0.152 & 26.3 & 587 & 0.327 & 17.8 \\
\hline $4 c$ & Opt3 & 1.594 & 76 & 1850 & 0.192 & 44.5 & 1437 & 0.393 & 20.9 \\
\hline \multicolumn{10}{|c|}{ Open Geometry Machines } \\
\hline $2 a$ & D20 PG002 & 2.41 & 81 & 132615 & 0.52 & 59.3 & 132122 & 1.81 & 4.3 \\
\hline
\end{tabular}




\begin{tabular}{|l|l|l|l|l|l|l|l|l|l|}
\hline 2b & D20 Ge311 & 2.41 & 81 & 22446 & 0.2 & 458.6 & 20755 & 0.66 & 37.9 \\
\hline $6 \mathrm{a}$ & Opt 1 & 1.54 & 81 & 10688 & 0.157 & 575.1 & 10009 & 0.343 & 250.5 \\
\hline $6 \mathrm{~b}$ & Opt 2 & 1.54 & 81 & 37842 & 0.246 & 337.8 & 37268 & 0.444 & 332.2 \\
\hline $6 \mathrm{c}$ & Opt 3 & 1.54 & 81 & 120495 & 0.385 & 179.3 & 126130 & 0.625 & 286.3 \\
\hline 8 & MMPD & 3.5 & 15 & 265362 & 0.0915 & $1.23 \times 10^{5}$ & 700509 & 1.438 & 56.7 \\
\hline $9 \mathrm{a}$ & HiRes detector mask & 1.54 & 81 & 15759 & 0.164 & 712.2 & 14389 & 0.358 & 303.4 \\
\hline $9 \mathrm{~b}$ & LoRes detector mask & 1.54 & 81 & 139873 & 0.352 & 297.9 & 141864 & 0.617 & 339.1 \\
\hline
\end{tabular}

$505 \quad$ Table 2: $\quad$ Summary of performance measures for simulations

506

507

508

\section{Appendix B: Instrument parameters used in the simulations}

This appendix presents parameters for the instruments modelled in the McSTAS and VITESS

509 simulations with the intention that they should permit duplication of the simulations described here.

510 D2B and D20 share the H11 beamtube at ILL and nearly all simulations use the following description.

511 The source is the sum of 3 Maxwellians with intensities $I_{\mathrm{i}}\left(\mathrm{n} \cdot \mathrm{cm}^{-2} \cdot \mathrm{s}^{-1}\right)$ corresponding to temperatures

$512 T_{\mathrm{i}}$ in Kelvin $\mathrm{I}_{1}=0.5874 \times 10^{13}, \mathrm{~T}_{1}=683.7 ; \mathrm{I}_{2}=2.5094 \times 10^{13}, \mathrm{~T}_{2}=257.7 ; \mathrm{I}_{3}=1.0343 \times 10^{12}, \mathrm{~T}_{3}=16.7$

513 Right handed Cartesian coordinates assume $x$ horizontal, $y$ vertical and $z$ along the neutron beam axis.

514 The in-pile beamtube has total length $5.1511 \mathrm{~m}$ and is modelled by a series of apertures at distance $\mathrm{Z}$

515 from the source

Source

$\mathrm{Z}=0$

$\mathrm{R}=0.11 \mathrm{~m}$

Win0

$Z=2.4621$

$\mathrm{R}=0.11 \mathrm{~m}$

Octagonal slit

$\mathrm{Z}=2.5121$

$0.15 \mathrm{~m}$ wide

519

520

$Z=2.5121$

$0.177 \mathrm{~m}$ wide rotated $45^{\circ}$

Win1

$\mathrm{Z}=3.5321$

$\mathrm{R}=0.0565$

Win2

$Z=4.0421$

$\mathrm{R}=0.0555$

Win3

$\mathrm{Z}=4.0871$

$\mathrm{R}=0.0575$

Win4

$Z=4.9991$

$\mathrm{R}=0.0575$

Win5

$Z=5.0061$

$\mathrm{R}=0.0625$

Win6

$\mathrm{Z}=5.1511$

$\mathrm{R}=0.0625$

The D2B monochromator is $16.05 \mathrm{~m}$ from the source and preceded by a $0.70 \mathrm{~m}$ long collimator at $15.705 \mathrm{~m}$ from the source. This monochromator position is used for all collimated instruments except 528 that in figure 3.

529 The D20 monochromator is $17.2 \mathrm{~m}$ from the source and preceded by an optional $0.257 \mathrm{~m}$ long 530 collimator at $16.4 \mathrm{~m}$ from the source. This monochromator position is used for all open geometry 531 instruments except that in figure 8.

532 All McSTAS simulations were run using Mn=1e8 and using SPLIT 5 at the monochromator and 533 SPLIT 10 at the sample.

534 In all cases except figure 5a, the sample was an $8 \mathrm{~mm}$ diameter NAC cylinder $2 \mathrm{~cm}$ high. In figure

$5355 \mathrm{a}$, the diameter was $2.5 \mathrm{~mm}$.

536 Collimated instruments were modelled in 2 parts. The $1^{\text {st }}$ part generated and saved a beam at a 537 distance $0.15 \mathrm{~m}$ after the sample at a scattering angle of $2 \theta_{\mathrm{s}}=-90^{\circ}$. This was used as the input to the $5382^{\text {nd }}$ part which modelled (usually) 128 collimator-detector pairs separated in $2 \theta_{\mathrm{S}}$ by 1.25 degrees. The $5392^{\text {nd }}$ part was run at 15 different angles separated by 5 ' to complete a scan. The final data was summed 
and intensities divided by a factor of 15 to compensate for the time division in stepping the detector bank.

Air attenuation was ignored in all simulations. No radial oscillating collimators were modelled.

\section{Existing Instruments - the baseline Figure 1 parameters: $\quad$ D2B as it is}

Vertically focussed Ge 533 monochromator; $I\left(\theta_{\mathrm{S}}\right)$ divided by 15 for detector steps

(a) $\theta_{\mathrm{M}}=67.5^{\circ} ; \lambda=1.594 \AA$

$$
\begin{array}{ll}
\left\{\alpha_{1} ; \eta_{\mathrm{M}} ; \alpha_{2} ; \alpha_{3}\right\}=\left\{5^{\prime} ; 12^{\prime} ; \text { Open } \approx 35^{\prime} ; 5^{\prime}\right\} & \left\{R_{\mathrm{M}} ; \tau_{1} ; \tau_{3}\right\}=\{0.40 ; 0.7 ; 0.5\} \\
\left\{L_{1} ; L_{2} ; L_{3}\right\} \quad=\{16.05 ; 2.645 ; 1.3\} & \left\{2 H_{\mathrm{M}} ; 2 H_{\mathrm{S}} ; 2 H_{\mathrm{D}}\right\}=\{0.30 ; 0.02 ; 0.10\} \\
\left\{L_{1 \_\mathrm{VH}} ; R_{\mathrm{MH}} ; L_{1} \_\mathrm{VV} ; R_{\mathrm{MV}}\right\}=\{\infty ; \infty ; 12.0 ; 4.005\} &
\end{array}
$$

The $\alpha_{3}$ collimators here are $0.30 \mathrm{~m}$ long, $0.30 \mathrm{~m}$ high and $0.015 \mathrm{~m}$ wide. The low transmission as measured is because of the very small $\alpha_{3}$. The $\alpha_{1}$ collimator is $0.70 \mathrm{~m}$ long and so is allocated a higher transmission.

(b) As for (a) but $2 H_{\mathrm{D}}=0.30 \mathrm{~m}$

(c) As for (a) but $\alpha_{1}=10^{\prime} ; \tau_{1}=0.8$

\section{Figure 2 parameters: $\quad$ D20 as it is}

(a) Vertically focussed PG002 monochromator; $\theta_{\mathrm{M}}=-21^{\circ} ; \lambda=2.41 \AA ; 0.05 \mathrm{~m}$ thick graphite filter

$$
\begin{aligned}
& \left\{\alpha_{1} ; \eta_{\mathrm{M}} ; \alpha_{2} ; \alpha_{3}\right\}=\left\{\text { Open } \approx 24^{\prime} ; 24^{\prime} ; \text { Open } \approx 42 ; \text { PSD 6' }\right\} \\
& \left\{R_{\mathrm{M}} ; \tau_{1} ; \tau_{3}\right\}=\{0.70 ; 1.0 ; 1.0\} \\
& \left\{L_{1} ; L_{2} ; L_{3}\right\}=\{17.2 ; 3.2 ; 1.47\} \\
& \left\{2 H_{\mathrm{M}} ; 2 H_{\mathrm{S}} ; 2 H_{\mathrm{D}}\right\}=\{0.30 ; 0.02 ; 0.15\} \\
& \left\{L_{1 \_\mathrm{VH}} ; R_{\mathrm{MH}} ; L_{1 \_\mathrm{Vv}} ; R_{\mathrm{MV}}\right\}=\{\infty ; \infty ; 13.15 ; 1.845\}
\end{aligned}
$$

(b) Vertically focussed Ge311 monochromator; $\theta_{\mathrm{M}}=-45^{\circ} ; \lambda=2.41 \AA$

$$
\begin{gathered}
\left\{\alpha_{1} ; \eta_{\mathrm{M}} ; \alpha_{2} ; \alpha_{3}\right\}=\left\{\text { Open } \approx 24^{\prime} ; 24^{\prime} ; \text { Open } \approx 42 ; \text { PSD } 6^{\prime}\right\} \quad\left\{R_{\mathrm{M}} ; \tau_{1} ; \tau_{3}\right\}=\{0.30 ; 1.0 ; 1.0\} \\
\left\{L_{1} ; L_{2} ; L_{3}\right\}=\{17.2 ; 3.2 ; 1.47\} \quad\left\{2 H_{\mathrm{M}} ; 2 H_{\mathrm{S}} ; 2 H_{\mathrm{D}}\right\}=\{0.30 ; 0.02 ; 0.15\} \\
\left\{L_{1 \_\mathrm{VH}} ; R_{\mathrm{MH}} ; L_{1 \_\mathrm{Vv}} ; R_{\mathrm{MV}}\right\}=\{\infty ; \infty ; 13.15 ; 3.64\}
\end{gathered}
$$

The value for $R_{\mathrm{M}}$ is lower here than for figure 1 because of the larger mosaic.

\section{Figure 3 parameters: $\quad$ Stride et afs CW PD}

Stride et al. showed using MC simulations that a given TOF PD is superior to a given CWPD by a factor between $\times 4$ and $\times 13$. Their CW PD model is simulated here for comparison to the optimized configurations which are more than a factor 13 better. Note that this does not prove an inherent superiority for either instrument type. The source used here is the ILL H11 Maxwellian. The beamtube is $6 \times 10 \mathrm{~cm}^{2}$ and the monochromators is a $20 \times 20 \mathrm{~cm}^{2}$ VFM.

Here 61 collimator-detector pairs are separated in $2 \theta_{\mathrm{S}}$ by 2.5 degrees. The $2^{\text {nd }}$ instrument part was run at 30 different angles separated by 5 ' to complete a scan. The final data were summed and intensities divided by a factor of 30 to compensate for the time division in stepping the detector bank. A test showed that using the $\mathrm{D} 2 \mathrm{~B}$ beamtube and monochromator position makes no difference to the data.

$$
\begin{aligned}
& \theta_{\mathrm{M}}=45^{\circ} ; \lambda=1.5 \AA \text { VFM Ge } 511 \\
& \left\{\alpha_{1} ; \eta_{\mathrm{M}} ; \alpha_{2} ; \alpha_{3}\right\}=\left\{12^{\prime} ; 12^{\prime} ; \text { Open; } 12^{\prime}\right\} \quad\left\{R_{\mathrm{M}}=0.30 ; \tau_{\alpha 1}=\tau_{\alpha 3}=0.85\right\}
\end{aligned}
$$

The value of $R_{\mathrm{M}}$ matches that used by Stride et al. but differs from that used in figure 1 and therefore the simulated detector intensities have been multiplied by $4 / 3$ to match the value $\left(R_{\mathrm{M}}=0.4\right)$ used elsewhere. $\tau_{\alpha 3}$ at 0.85 is much larger than the transmission for comparable collimators modelled elsewhere in this work so that intensities have also been multiplied by 0.7 / 0.85 to compensate.

$$
\left\{L_{1} ; L_{2} ; L_{3}\right\}=\{10 ; 2.0 ; 1.5\} \quad\left\{2 H_{\mathrm{M}} ; 2 H_{\mathrm{S}} ; 2 H_{\mathrm{D}}\right\}=\{0.20 ; 0.02 ; 0.10\}
$$


608

609

610

611

612

613

614

615

616

617

618

619

620

621

622

623

624

625

626

627

$61\left(2.54 \times 10 \mathrm{~cm}^{2} \mathrm{~W} \times \mathrm{H}\right)$ detectors with $12^{\prime}$ collimators $2.5^{\circ}$ apart covering $150^{\circ}$ and with $30 \times 0.0833^{\circ}$ steps. Note that the $2 \theta_{\mathrm{S}}$ range here is smaller than in figure 1 . Here the intensities are increased but the peak widths $A_{1 / 2}$ increase sharply above $2 \theta_{\mathrm{S}}=45^{\circ}$, a consequence of the small $\theta_{\mathrm{M}}$ used with a horizontally flat monochromator.

\section{Optimized Instruments - (Optimized for samples of cubic structure)}

\section{Figure 4 parameters: $\quad$ Optimized CW PD using collimators and VFM}

\section{For all three models}

Ge 533 VFM; $\theta_{\mathrm{M}}=67.5^{\circ} ; \lambda=1.594 \AA$ 品 $I\left(\theta_{\mathrm{S}}\right)$ divided by 15 for detector steps

$\left\{L_{1} ; L_{2} ; L_{3}\right\} \quad=\{16.05 ; 2.645 ; 1.3\} \quad\left\{L_{1 \_\mathrm{VH}} ; R_{\mathrm{MH}} ; L_{1 \_\mathrm{VV}} ; R_{\mathrm{MV}}\right\}=\{\infty ; \infty ; 12.0 ; 4.005\}$

(a) $\quad\left\{\alpha_{1} ; \eta_{\mathrm{M}} ; \alpha_{2} ; \alpha_{3}\right\}=\left\{10.23^{\prime} ; 6.86^{\prime} ; 10.43^{\prime} ; 5^{\prime}\right\} \quad\left\{R_{\mathrm{M}} ; \tau_{\alpha 1} ; \tau_{\alpha 2} ; \tau_{\alpha 3}\right\}=\{0.40 ; 0.85 ; 0.7 ; 0.5\}$ Because the flight paths themselves provide some collimation, to achieve a FWHM of 10.23' requires an $\alpha_{1}$ Soller collimator of FWHM 11.6'. Similarly, the $\alpha_{2}$ collimator modelled had FWHM 10.92'. $\left\{2 H_{\mathrm{M}} ; 2 H_{\mathrm{S}} ; 2 H_{\mathrm{D}}\right\}=\{0.22 ; 0.02 ; 0.10\}$

(b) $\quad\left\{\alpha_{1} ; \eta_{\mathrm{M}} ; \alpha_{2} ; \alpha_{3}\right\}=\left\{16.23^{\prime} ; 10.90^{\prime} ; 16.55^{\prime} ; 7.94^{\prime}\right\} \quad\left\{R_{\mathrm{M}} ; \tau_{\alpha 1} ; \tau_{\alpha 2} ; \tau_{\alpha 3}\right\}=\{0.40 ; 0.9 ; 0.8 ; 0.65\}$ The simulations used Soller collimators of FWHM $\alpha_{1}=24^{\prime} ; \alpha_{2}=18.8^{\prime}$ $\left\{2 H_{\mathrm{M}} ; 2 H_{\mathrm{S}} ; 2 H_{\mathrm{D}}\right\}=\{0.278,0.02,0.127\}$

(c) $\quad\left\{\alpha_{1} ; \eta_{\mathrm{M}} ; \alpha_{2} ; \alpha_{3}\right\}=\left\{20.46^{\prime} ; 13.72^{\prime} ; 20.86^{\prime} ; 10^{\prime}\right\}\left\{R_{\mathrm{M}} ; \tau_{\alpha 1} ; \tau_{\alpha 2} ; \tau_{\alpha 3}\right\}=\{0.40 ; 1.0 ; 0.85 ; 0.7\}$ The simulations used a Soller collimator $\alpha_{2}$ of FWHM $\alpha_{2}=26^{\prime}$ and an open beamtube for $\alpha_{1}$ giving a FWHM of about 22'. $\left\{2 H_{\mathrm{M}} ; 2 H_{\mathrm{S}} ; 2 H_{\mathrm{D}}\right\}=\{0.312,0.02,0.142\}$

\section{Figure 5 parameters: $\quad$ Optimized CW PD using open geometry and DFM}

Ge 533 DFM; $\theta_{\mathrm{M}}=-45^{\circ} ; \lambda=1.594 \AA$
(a) $\left\{\eta_{\mathrm{M}} ; \alpha_{3}\right\}=\left\{12^{\prime} ; 6^{\prime} \mathrm{PSD}\right\}$
$\left\{\alpha_{1} ; \alpha_{2}\right\}=\left\{4.14^{\prime} ; 12.25^{\prime}\right\}$
$\left\{R_{\mathrm{M}}\right\}=\{0.40\}$

The detector is a 1600 wire PSD of radius $1.47 \mathrm{~m}$ with wire spacing $2.56 \mathrm{~mm}$ giving a 6 ' angular separation. We expected that the effective $\alpha_{3}$ collimation should include sample and detector widths, $2 W_{\mathrm{S}}$ and $W_{\mathrm{D}}$, so that $\alpha_{3} \approx \operatorname{atan}\left\{\left(2 W_{\mathrm{S}}+W_{\mathrm{D}}\right) / 2 L_{3}\right\}$

$$
\begin{array}{ll}
\left\{L_{1} ; L_{2} ; L_{3}\right\}=\{17.2 ; 3.2 ; 1.47\} & \left\{L_{1} \mathrm{VH}_{1} ; R_{\mathrm{MH}} ; L_{1 \_\mathrm{VV}} ; R_{\mathrm{MV}}\right\}=\{7.31 ; 6.3 ; 13.15 ; 3.64\} \\
\left\{2 H_{\mathrm{M}} ; 2 H_{\mathrm{S}} ; 2 H_{\mathrm{D}}\right\}=\{0.242 ; 0.02 ; 0.110\} & \left\{2 \mathrm{~W}_{\mathrm{V}} ; 2 \mathrm{~W}_{\mathrm{M}} ; 2 \mathrm{~W}_{\mathrm{S}}\right\}=\{0.0124 ; 0.0141 ; 0.0025\}
\end{array}
$$

(b) As for (a) but $2 \mathrm{~W}_{\mathrm{S}}=0.008 \quad$ so effectively $\left\{\alpha_{2} ; \alpha_{3}\right\}=\left\{11.9^{\prime} ; 12.3^{\prime}\right\}$

\section{Figure 6 parameters: $\quad$ Open geometry DFM CW PD Optimized at various resolutions}

All figure 6 simulations use a "Ge 511 ” DFM at $\theta_{\mathrm{M}}=-45^{\circ}, \lambda=1.540 \AA$ and $\quad\left\{L_{1}, L_{2}, L_{3}\right\}=\{17.2,3.0,1.5\} \quad\left\{L_{1 \_\mathrm{VH}}, L_{1-\mathrm{Vv}}, R_{\mathrm{MH}}, R_{\mathrm{MV}}\right\}=\{6.850,13.15,5.90,3.455\}$ There is flexibility in the optimal choice of $\eta_{\mathrm{M}} / \alpha_{1}$ and this was used to set the monochromator mosaic to 12 ' (to match that used now on D2B) and a value of $R_{\mathrm{M}}=0.40$ was assumed. The detector is a 1600 wire PSD of radius $1.47 \mathrm{~m}$ with wire spacing $2.56 \mathrm{~mm}$ giving a 6 ' angular separation. This instrument is the same for figures $6 a, 6 b$ and $6 \mathrm{c}$ with adjusted slit widths. 
(a) Here the parameters were chosen to give a value for $R_{\mathrm{P}_{-} \mathrm{RMS}}$ of 0.157 to match that for figure $1 \mathrm{a}$. Therefore,

$$
\begin{aligned}
& \left\{\left(4 \alpha_{1}^{-2}+\eta_{\mathrm{M}}{ }^{-2}\right)^{-1 / 2}, \alpha_{2} \alpha_{3}\right\}=\left\{2.593^{\prime}, 13.59^{\prime}, 7.64^{\prime}\right\} \quad \begin{array}{c}
\phi=0.0427=2.45^{\circ} \\
\left\{2 H_{\mathrm{M}}, 2 H_{\mathrm{S}}, 2 H_{\mathrm{D}}\right\}=\{0.256,0.02,0.127\}
\end{array}\left\{2 W_{\mathrm{V}}, 2 W_{\mathrm{M}}, 2 W_{\mathrm{S}}\right\}=\{0.0150,0.0168,0.008\}
\end{aligned}
$$

The slit widths were adjusted by factors of $\sqrt{ } 2$ to allow for the rectangular variation of transmission with angular divergence expected for the open beam tubes used here. The optimisation requires that $\left(4 \alpha_{1}^{-2}+\eta_{\mathrm{M}}{ }^{-2}\right)^{-1 / 2}=2.593$ ', so setting $\eta_{\mathrm{M}}=12^{\prime}$ gives $\alpha_{1}=5.31$ ' and $2 W_{\mathrm{V}}=\left(\sqrt{ } 2 \bullet \alpha_{1} \bullet L_{1 \_\mathrm{VH}}\right)=0.015 \mathrm{~m}$ $\alpha_{2}=13.59^{\prime}$ giving $2 W_{\mathrm{M}}=\left(\sqrt{ } 2 \bullet \alpha_{2} \bullet L_{2}\right)=0.0168$

(b) Optimised medium resolution CWPD. Here the optimisation parameters were derived assuming that $\alpha_{3}=12$ '

$$
\begin{aligned}
& \left\{\left(4 \alpha_{1}{ }^{-2}+\eta_{\mathrm{M}}{ }^{-2}\right)^{-1 / 2}, \alpha_{2} \alpha_{3}\right\}=\left\{4.077^{\prime}, 21.37^{\prime}, 12^{\prime}\right\} \quad \quad \quad \phi=0.0536=3.07^{\circ} \\
& \left\{2 H_{\mathrm{M}}, 2 H_{\mathrm{S}}, 2 H_{\mathrm{D}}\right\}=\{0.321,0.02,0.160\} \quad\left\{2 W_{\mathrm{V}}, 2 W_{\mathrm{M}}, 2 W_{\mathrm{S}}\right\}=\{0.0244,0.0264,0.008\} \\
& \left(4 \alpha_{1}^{-2}+\eta_{\mathrm{M}}^{-2}\right)^{-1 / 2}=4.077^{\prime} \text { Choosing } \eta_{\mathrm{M}}=12^{\prime} \text { gives } \alpha_{1}=8.67
\end{aligned}
$$

This instrument is the same as that for figure $6 \mathrm{a}$ with the only differences being adjusted slit widths.

(c) Optimised high intensity CWPD. Here the optimisation parameters were derived assuming that $\alpha_{3}=18^{\prime}$. This corresponds to $2 H_{\mathrm{D}}=0.20$

$$
\begin{aligned}
& \left\{\left(4 \alpha_{1}{ }^{-2}+\eta_{\mathrm{M}}{ }^{-2}\right)^{-1 / 2}, \alpha_{2} \alpha_{3}\right\}=\left\{6.37^{\prime}, 33.4^{\prime}, 18.75^{\prime}\right\} \\
& \left\{2 H_{\mathrm{M}}, 2 H_{\mathrm{S}}, 2 H_{\mathrm{D}}\right\}=\{0.401,0.02,0.200\} \quad\left\{2 W_{\mathrm{V}}, 2 W_{\mathrm{M}}, 2 W_{\mathrm{S}}\right\}=\{0.0424,0.0412,0.008\} \\
& \left\{4 \alpha_{1}^{-2}+\eta_{\mathrm{M}}{ }^{-2}\right\}^{-1 / 2}=6.37^{\prime} \text { and choosing } \eta_{\mathrm{M}}=12^{\prime} \text { gives } \alpha_{1}=15.05^{\prime} .
\end{aligned}
$$

\section{Figure 7 parameters: Open geometry DFM CW PD Optimized for small $2 \theta_{S} / d_{S}$ range}

(a) "Ge511" DFM with $\theta_{\mathrm{M}}=-45^{\circ} ; \lambda=1.594 \AA$

The optimisation was applied here over a scattering angle range $80^{\circ}<2 \theta_{\mathrm{S}}<100^{\circ}$

Here a Soller collimator is used to define the beam angular spread before the monochromator

$$
\begin{array}{ll}
\left\{\alpha_{1} ; \eta_{\mathrm{M}} ; \alpha_{3}\right\}=\left\{6.4^{\prime} ; 12^{\prime} ; 6^{\prime} \mathrm{PSD}\right\} & \left\{R_{\mathrm{M}} ; \tau_{\alpha 1}\right\}=\{0.40 ; 0.75\} \\
\left\{L_{1} ; L_{2} ; L_{3}\right\}=\{17.2 ; 3.2 ; 1.47\} & \left\{L_{1 \_\mathrm{VH}} ; R_{\mathrm{MH}} ; L_{1 \_\mathrm{VV}} ; R_{\mathrm{MV}}\right\}=\{\infty ; 9.05 ; 13.15 ; 3.64\} \\
\left\{2 H_{\mathrm{M}} ; 2 H_{\mathrm{S}} ; 2 H_{\mathrm{D}}\right\}=\{0.299 ; 0.02 ; 0.136\} & \left\{2 \mathrm{~W}_{\mathrm{V}} ; 2 \mathrm{~W}_{\mathrm{M}} ; 2 \mathrm{~W}_{\mathrm{S}}\right\}=\{0.2 ; 0.0789 ; 0.008\}
\end{array}
$$

(b) PG002 DFM with $\theta_{\mathrm{M}}=-21^{\circ} ; \lambda=2.41 \AA \quad\left\{R_{\mathrm{M}}\right\}=\{0.70\}$

The optimisation was applied here over a sample $d$-spacing range $2 \AA<d_{\mathrm{S}}<3 \AA$

$$
\begin{aligned}
& \left\{\eta_{\mathrm{M}} ; \alpha_{3}\right\}=\left\{24^{\prime} ; 6^{\prime} \mathrm{PSD}\right\} \quad\left\{L_{1} ; L_{2} ; L_{3}\right\}=\{17.2 ; 3.2 ; 1.47\} \\
& \left\{L_{1 \_\mathrm{VH}} ; R_{\mathrm{MH}} ; L_{1 \_\mathrm{Vv}} ; R_{\mathrm{MV}}\right\}=\{8.48 ; 12.97 ; 13.15 ; 1.84\} \\
& \left\{2 H_{\mathrm{M}} ; 2 H_{\mathrm{S}} ; 2 H_{\mathrm{D}}\right\}=\{0.26 ; 0.02 ; 0.118\} \quad\left\{2 \mathrm{~W}_{\mathrm{V}} ; 2 \mathrm{~W}_{\mathrm{M}} ; 2 \mathrm{~W}_{\mathrm{S}}\right\}=\{0.0134 ; 0.0535 ; 0.008\}
\end{aligned}
$$

\section{Figure 8 parameters: $\quad$ Open geometry DFM MMPD optimized for $\kappa<3.8 \AA^{-1}$}

This instrument is sited on the ILL horizontal cold source tube $\mathrm{H} 5$; radius $=0.21$

$$
\mathrm{I}_{1}=1.022 \times 10^{13}, \mathrm{~T}_{1}=413.5 ; \quad \mathrm{I}_{2}=3.44 \times 10^{13}, \mathrm{~T}_{2}=145.8 ; \mathrm{I}_{3}=2.78 \times 10^{13}, \mathrm{~T}_{3}=40.1 \mathrm{~K}
$$

The layout is simplified: Source; $2.155 \mathrm{~m}$ gap

The monochromator is $1 \mathrm{~m}$ from the end of a $16.313 \mathrm{~m}$ long, $6 \times 20 \mathrm{~cm}^{2}, \mathrm{~m}=2$ guide.

$$
\text { PG002 DFM } \theta_{\mathrm{M}}=-31.4^{\circ} ; \lambda=3.5 \AA ; \quad\left\{R_{\mathrm{M}}\right\}=\{0.80\}
$$

$\left\{\alpha_{1} ; \eta_{\mathrm{M}} ; \alpha_{3}\right\}=\left\{\right.$ Open (ie guide so $\left.\pm 21^{\prime}\right) ; 24^{\prime} ; 6^{\prime} 1601$ wire PSD $\}$

$\left\{L_{1}\right\}\left\{L_{2} ; L_{3}\right\}=\{2.5+15+1.0\}\{2.5 ; 1.5\} \quad\left\{L_{1 \_\mathrm{VH}} ; R_{\mathrm{MH}} ; L_{1 \_\mathrm{Vv}} ; R_{\mathrm{MV}}\right\}=\{\infty ; 3.972 ; \infty ; 2.605\}$

$\left\{2 H_{\mathrm{M}} ; 2 H_{\mathrm{S}} ; 2 H_{\mathrm{D}}\right\}=\{0.20 ; 0.02 ; 0.18\} \quad\left\{2 \mathrm{~W}_{\mathrm{V}} ; 2 \mathrm{~W}_{\mathrm{M}} ; 2 \mathrm{~W}_{\mathrm{S}}\right\}=\{0.06 ; 0.06 ; 0.008\}$ 
675 Figure 9 parameters: Optimized Open geometry DFM CW PD - Orange Peel

676 detector mask

(a) “Ge 511” DFM $\left\{\theta_{\mathrm{M}}, \lambda, R_{\mathrm{M}}\right\}=\left\{-45^{\circ}, 1.54 \AA\right.$ A, 0.40$\} \phi_{2}=0.0423=2.42^{\circ} \quad \phi_{3 \_90}=0.0719=4.12^{\circ}$ 1601 wire banana detector $10^{\circ}<2 \theta_{\mathrm{S}}<170^{\circ} \quad 3.33 \mathrm{~mm}$ spacing, $8^{\prime}$ collimation

679

$\left\{L_{1}, L_{2}, L_{3}\right\}=\{17.2,3.0,1.5\}$

$\left\{2 H_{\mathrm{M}}, 2 H_{\mathrm{S}}, 2 H_{\mathrm{D} \_90}\right)=\{0.253,0.02,0.215\}$

680 $\left\{\left(4 \alpha_{1}^{-2}+\eta_{\mathrm{M}}^{-2}\right)^{-1 / 2}, \alpha_{2} \alpha_{3}\right\}=\left\{2.72^{\prime}, 14.24^{\prime}, 8^{\prime}\right\}$

681

$\left\{L_{1 \_\mathrm{VH}}, L_{1 \_\mathrm{VV}}, R_{\mathrm{MH}}, R_{\mathrm{MV}}\right\}=\{6.85,13.15,5.901,3.455\} \quad\left\{2 W_{\mathrm{V}}, 2 W_{\mathrm{M}}, 2 W_{\mathrm{S}}\right\}=\{0.0157,0.0176,0.008\}$

682

Choose $\eta_{\mathrm{M}}=12$ ' so

$$
\begin{aligned}
& \alpha_{1}=5.58^{\prime} \text { and } 2 W_{\mathrm{V}}=\left(\sqrt{ } 2 \bullet \alpha_{1} \bullet L_{1_{1} \mathrm{VH}}\right)=0.0157 . \\
& \alpha_{2}=14.24^{\prime} \text { so } 2 W_{\mathrm{M}}=\left(\sqrt{ } 2 \bullet \alpha_{2} \bullet L_{2}\right)=0.0176
\end{aligned}
$$

684

685

(b) As for figure 9a but

686

687

$\left\{\left(4 \alpha_{1}^{-2}+\eta_{\mathrm{M}}^{-2}\right)^{-1 / 2}, \alpha_{2} \alpha_{3}\right\}=\left\{6.11^{\prime}, 32.05^{\prime}, 18^{\prime}\right\}$

$\phi_{2}=0.0635=3.64^{\circ} \quad \phi_{3 \_90}=0.108=6.18^{\circ}$

And again choosing $\eta_{\mathrm{M}}=12$ '

688

$\left\{2 W_{\mathrm{V}}, 2 W_{\mathrm{M}}, 2 W_{\mathrm{S}}\right\}=\{0.040,0.0396,0.008\}$

$\left\{2 H_{\mathrm{M}}, 2 H_{\mathrm{S}}, 2 H_{\mathrm{D}_{-} 90}\right)=\{0.380,0.02,0.323\}$

689 

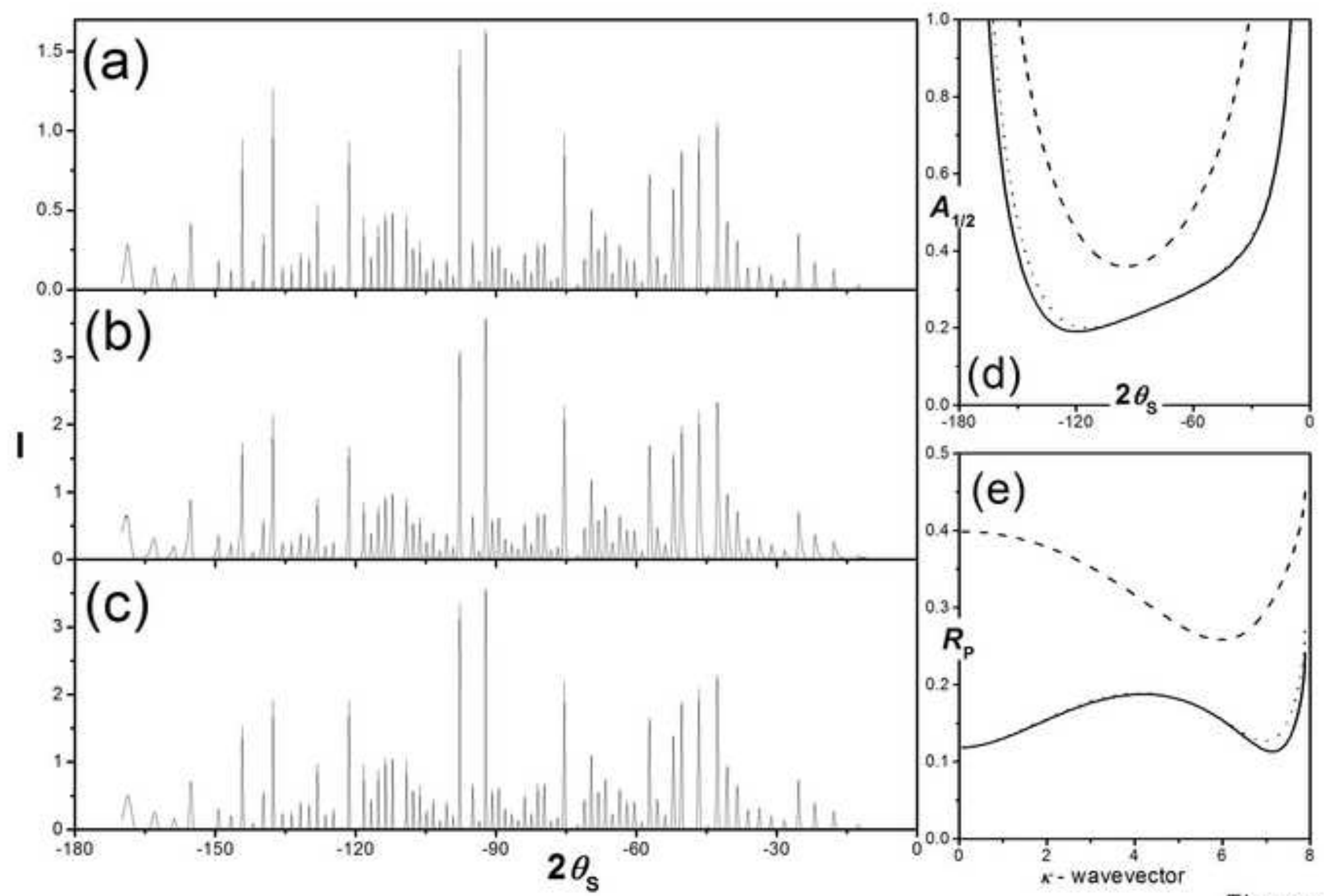

Figure 1 

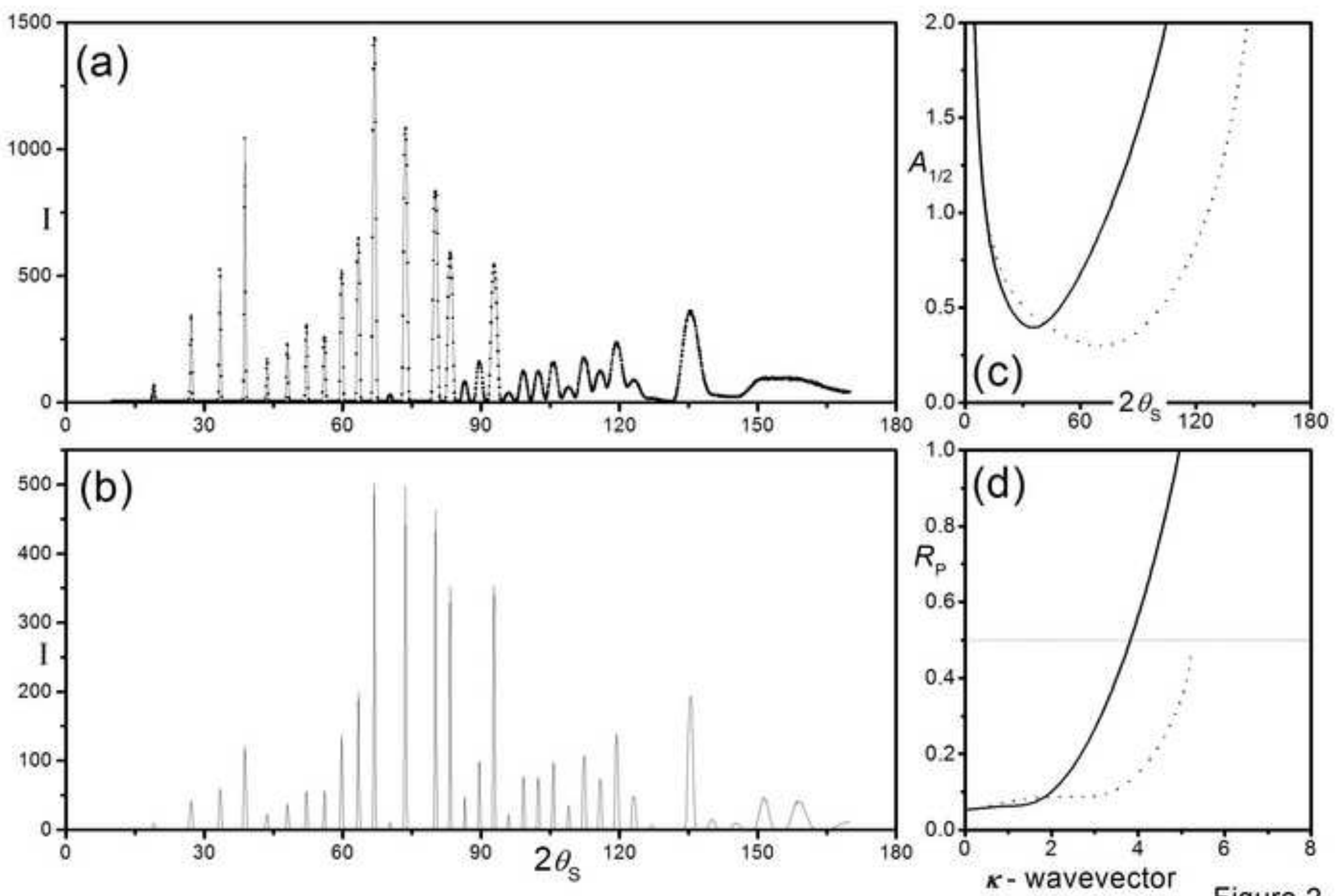

Figure 2 

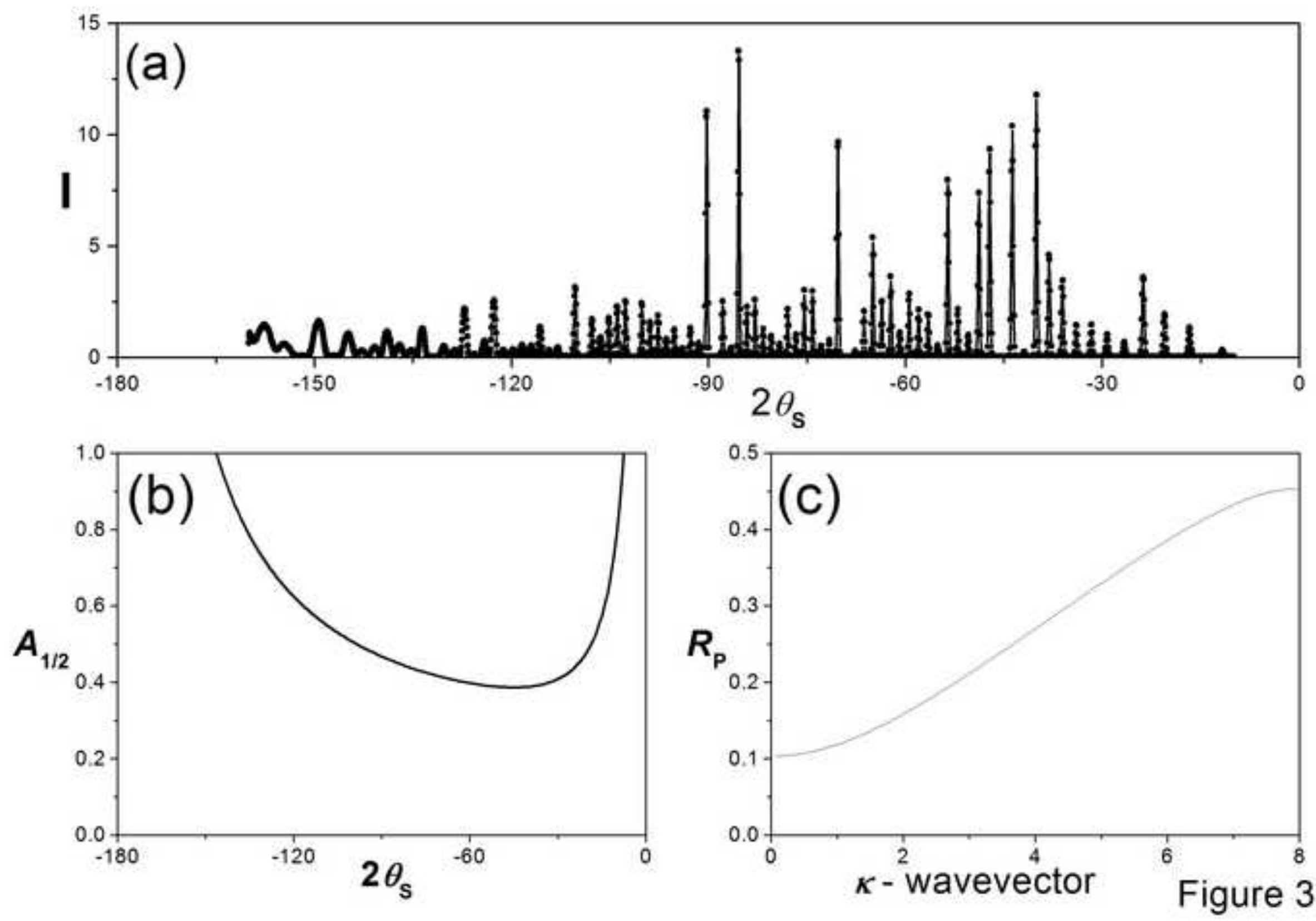

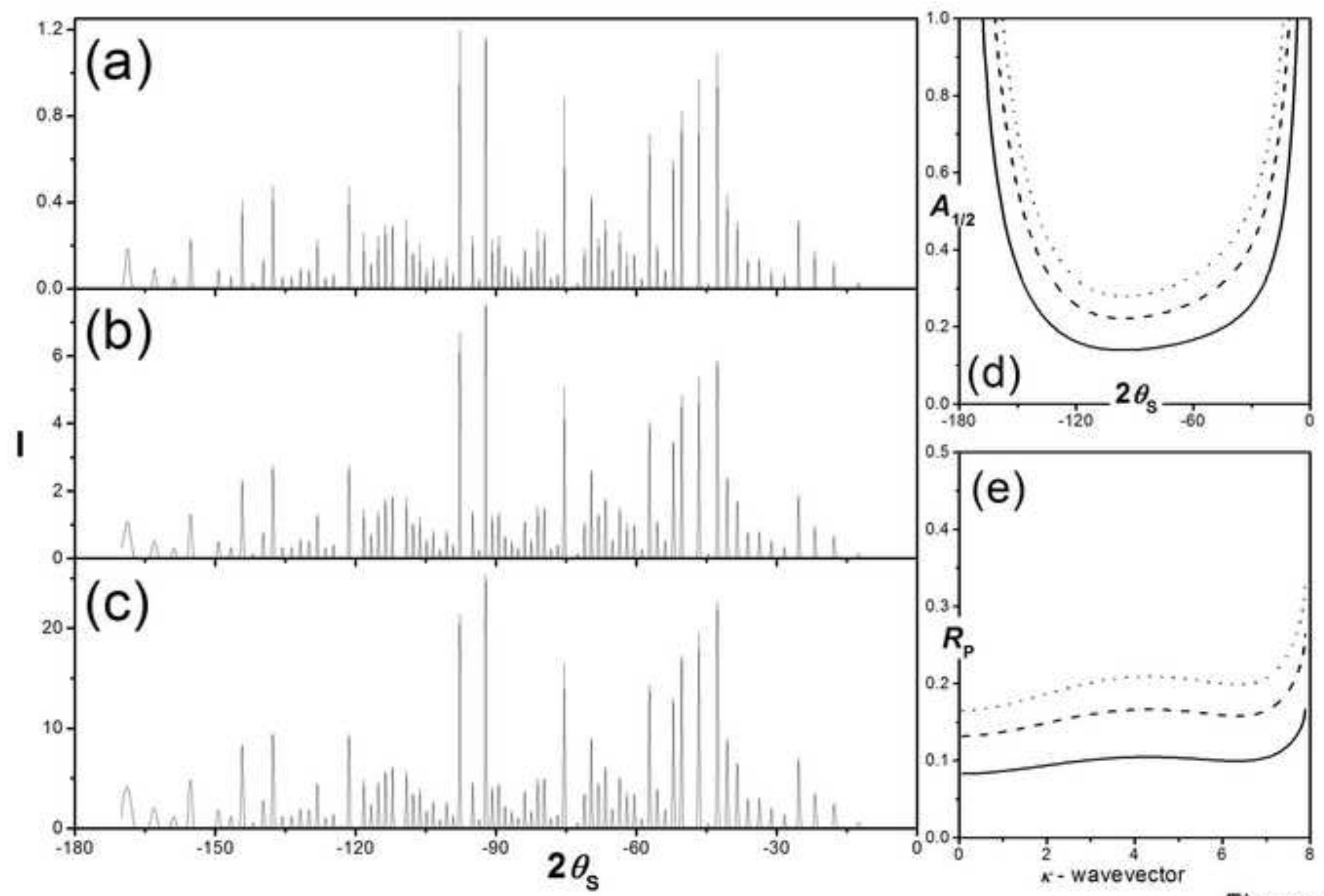

Figure 4 

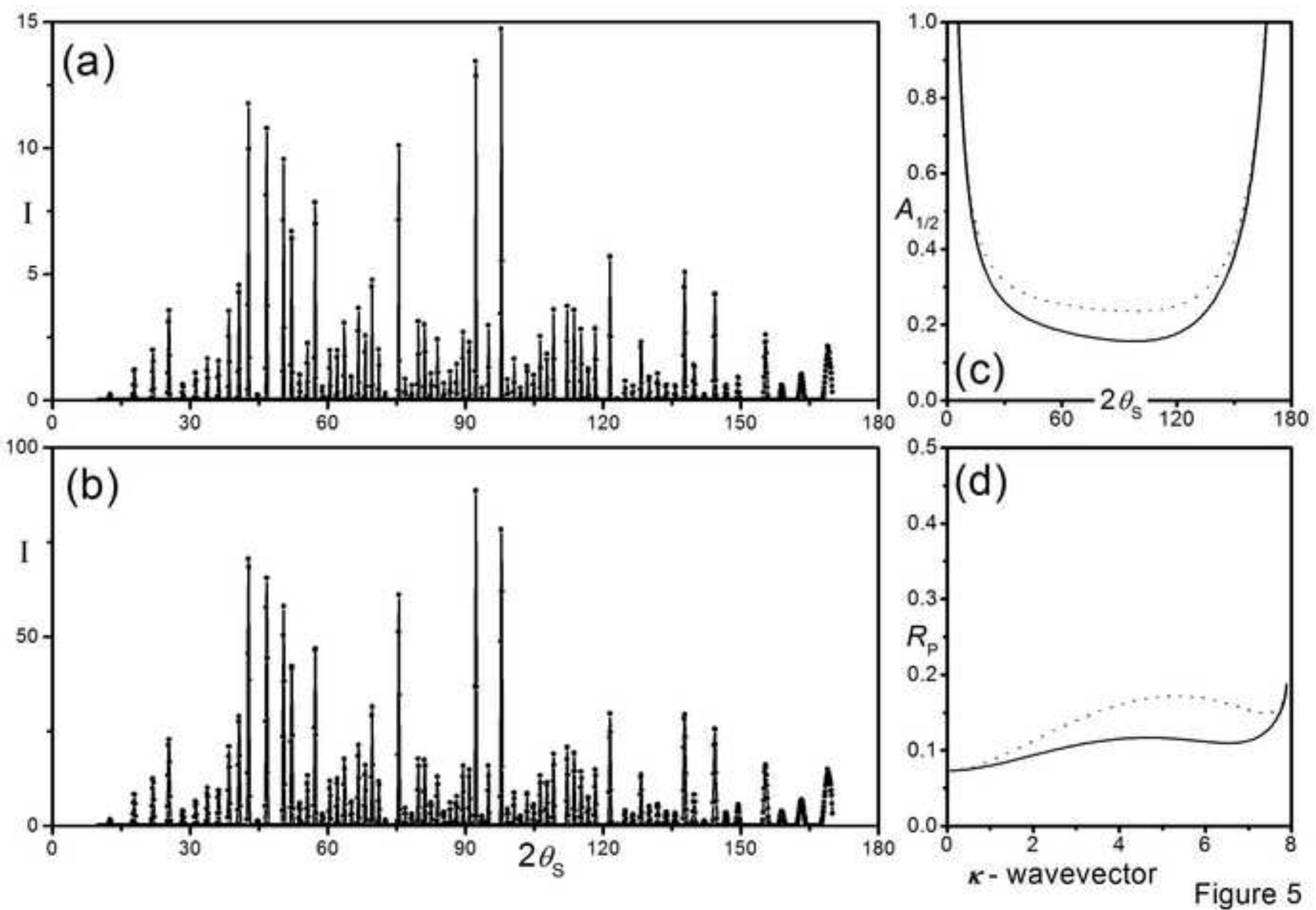

Figure 5 

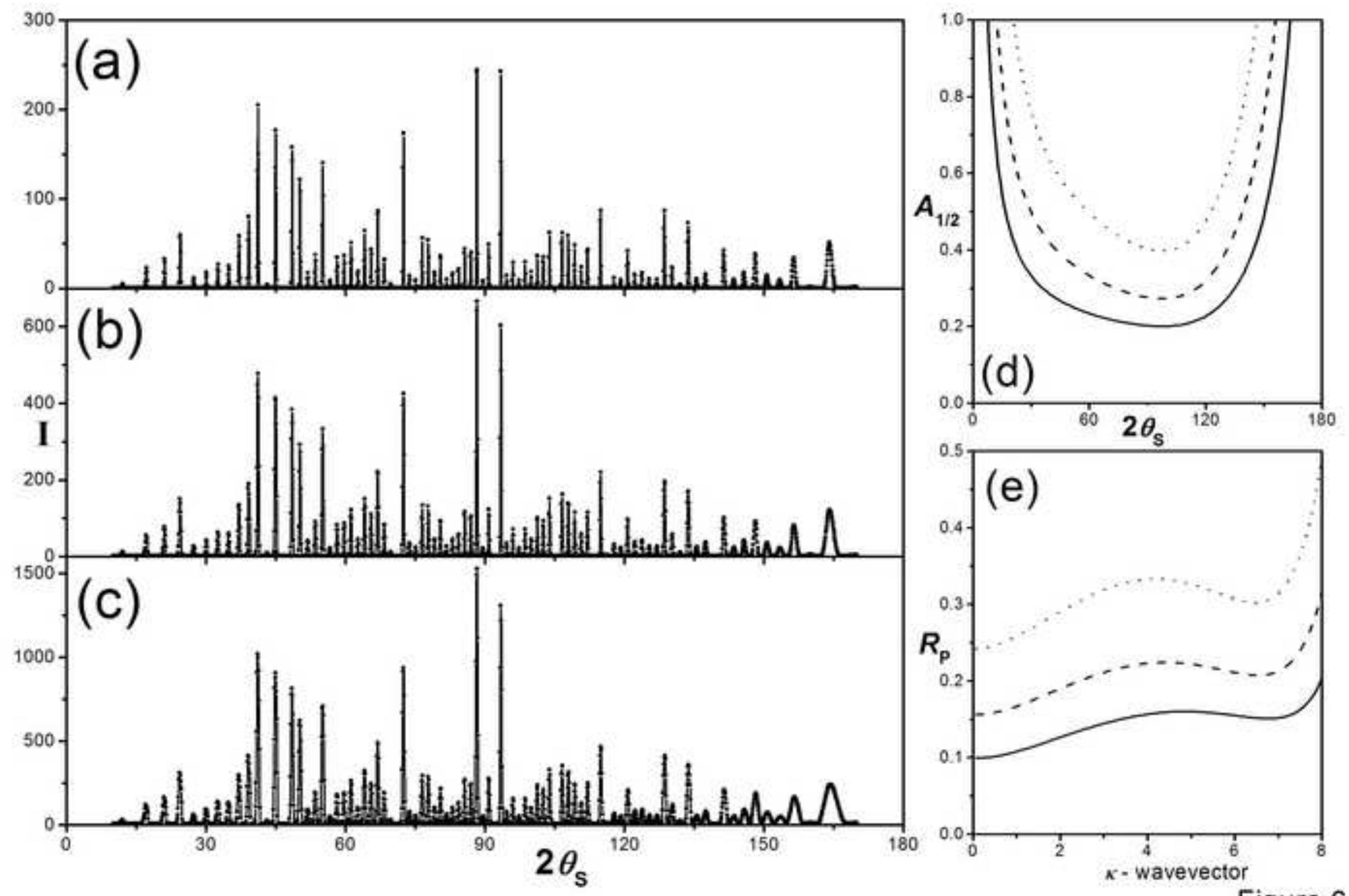

Figure 6 

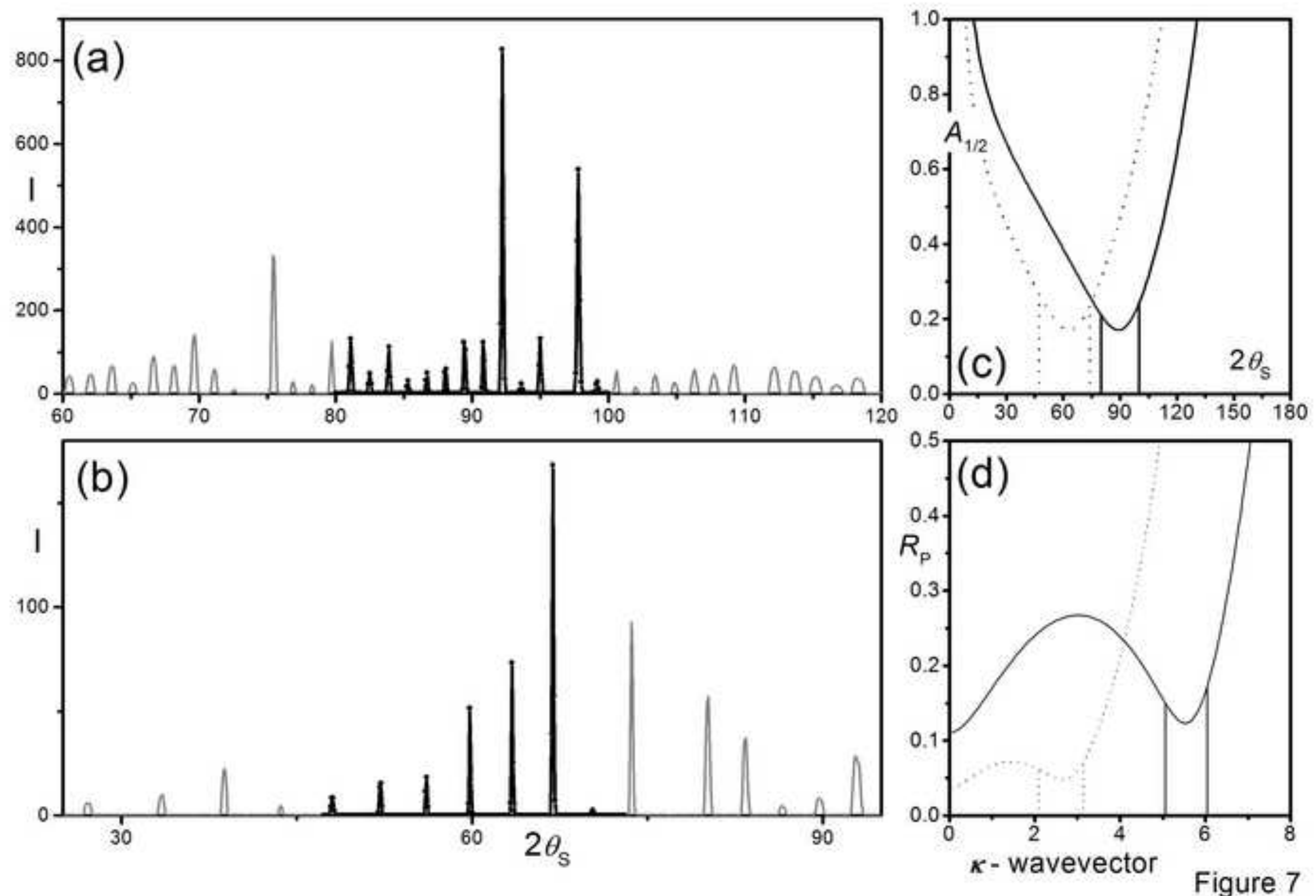

Figure 7 

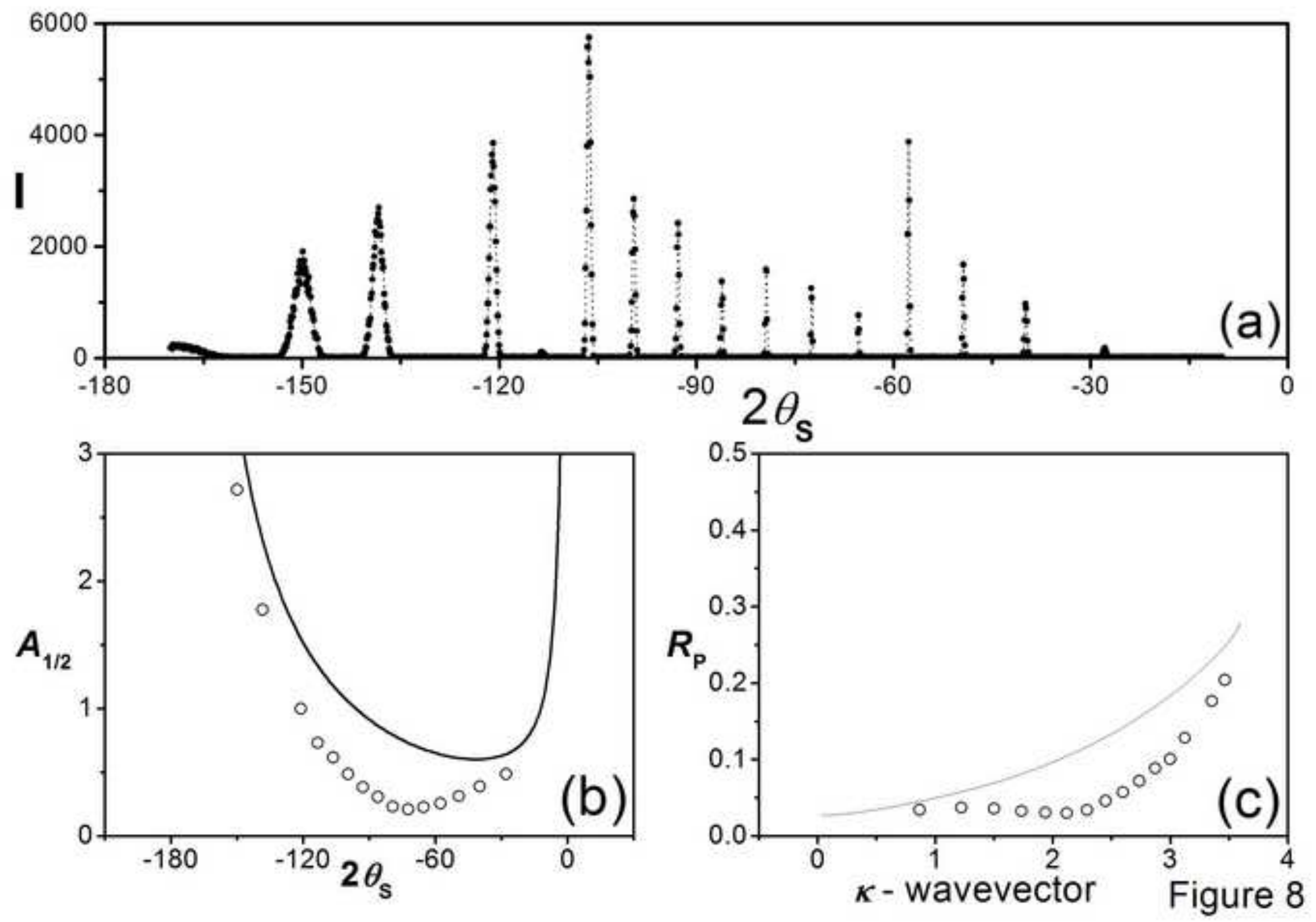

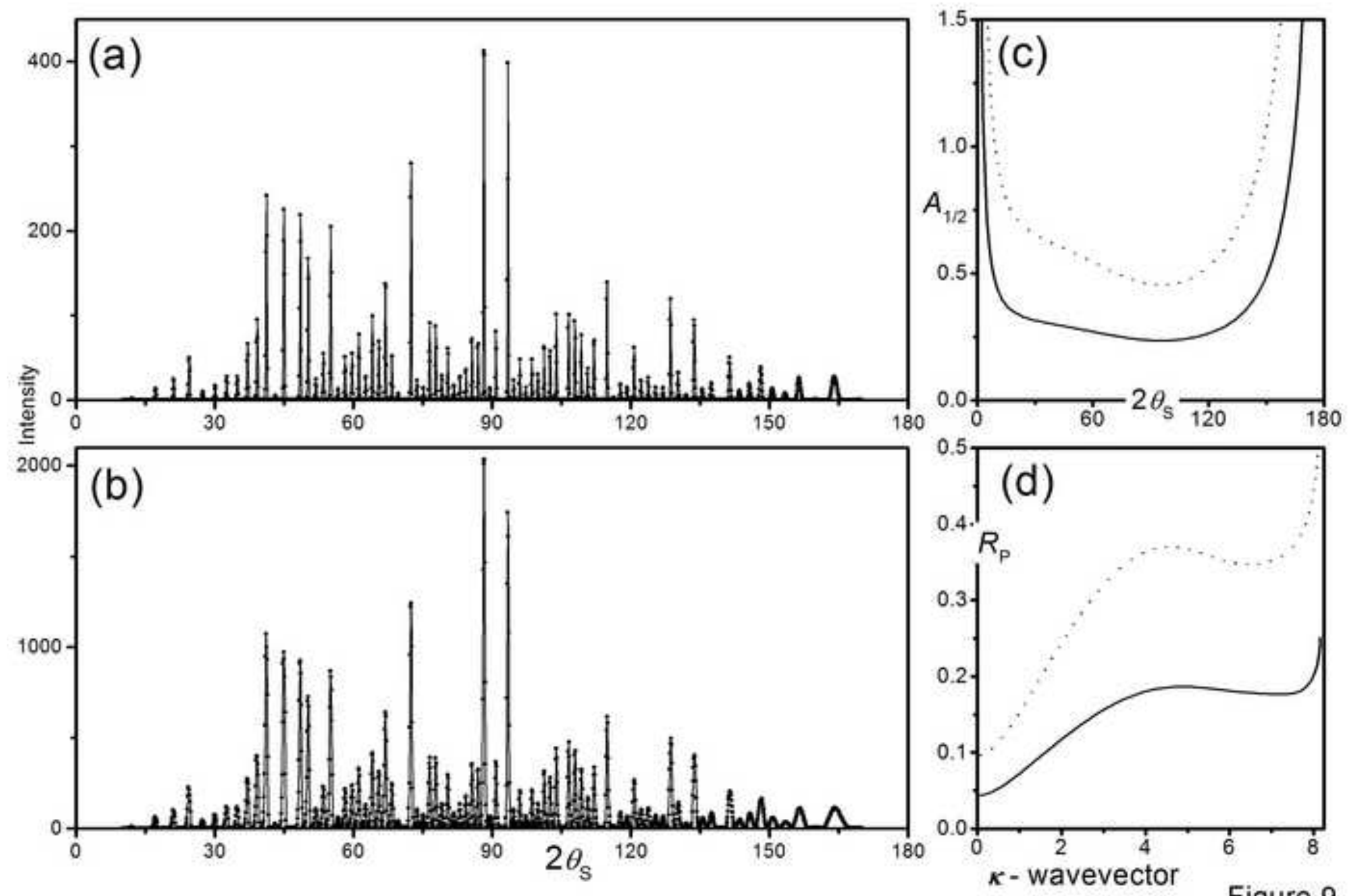

Figure 9 